\title{
Characterizations and Classifications of Argumentative Entailments
}

\author{
Ofer Arieli $^{1}$, AnneMarie Borg ${ }^{2}$, Christian Straßer ${ }^{3}$ \\ ${ }^{1}$ School of Computer Science, Tel-Aviv Academic College, Israel \\ ${ }^{2}$ Department of Information and Computing Sciences, Utrecht University, The Netherlands \\ ${ }^{3}$ Institute for Philosophy II, Ruhr University Bochum, Germany \\ oarieli@mta.ac.il, a.borg@uu.nl, christian.strasser@rub.de
}

\begin{abstract}
In this paper we provide a detailed analysis of the inference process induced by logical argumentation frameworks. The frameworks may be defined with respect to any propositional language and logic, different arguments that represent deductions in the logic, various support-based attack relations between arguments, and all the complete Dung-style semantics for the frameworks. We show that, ultimately, for characterizing the inference process with respect to a given framework, extension-based semantics may be divided into two types: single-extension and multiple-extension, which induce respective kinds of entailment relations. These entailments are further classified by the way they tolerate new information (nonmonotonicity-related properties) and maintain conflicts among arguments (inconsistency-related properties).
\end{abstract}

\section{Introduction}

Since its introduction, formal argumentation has become a core study in AI (Bench-Capon and Dunne 2007) and has been used in several real-life applications (Atkinson et al. 2017; Cerutti et al. 2017). It models reasoning processes by argumentation frameworks, consisting of arguments and attack relations between them (Dung 1995). In this context, arguments may be logical expressions based on an underlying logic (i.e., language and inference relation), a knowledge-base, and a set of rules, in which case the attacks are derived from the structure of the arguments (see (Besnard et al. 2014; Besnard and Hunter 2018)). Conclusions are derived by determining the extensions of the framework: sets of arguments that can collectively be considered as acceptable, under different semantics.

It is evident that the nature of argumentation frameworks, and in particular those that are based on the logical settings described above (called logical or deductive argumentation; (Besnard and Hunter 2018)), is affected by several factors, including the language and the deductive base of the arguments, the way arguments attack each other, the argumentative semantics, and the method of choosing the conclusions from the extensions (credulous or skeptical choices). In this paper we provide an analysis on how the inference properties of argumentation frameworks are determined by their above-mentioned ingredients.

A variety of formal properties have been introduced for evaluating the plausibility of argumentation frameworks (see, e.g., (Caminada 2018)), most of them are extensively studied in the literature with respect to different forms of (logical) argumentation frameworks (see the next section for some discussion and references). In this paper, we concentrate on the properties that refer to the entailment relations that are induced by the argumentation frameworks. This, together with some well-known postulates from the literature of non-monotonic and paraconsistent reasoning (e.g., (Rescher and Manor 1970; Kraus, Lehmann, and Magidor 1990)), allow us to classify and characterize a variety of argumentative entailments and thus analyze the conclusion making paradigms in logical argumentation. Some of the new results reported in this paper are the following:

1. We show that, despite the variety of Dung-style semantics for logical argumentation frameworks, once the other ingredients (namely, the base logic, the types of attacks and the extension selection method) are fixed, inferences are determined according to only one factor: whether the semantics results in a single extension or, possibly, multiple extensions. As we shall see in the sequel, this has far reaching consequences on the reasoning process.

2. From a different angle, we observe that (once the underlying semantics is determined) the nature of the attack rules has a crucial effect on the properties of the entailment relations. Here, the main factor is whether the attack is on a particular assumption of an argument (direct attacks) or on a set of assumptions (set attacks).

3. We show how the extension selection method, namely whether any extension or all the extensions are taken into consideration, affects the inference process.

We start in Section 2 by some references to related works. Then, in Sections 3 and 4 we recall some basic notions from logical argumentation and define the scope of our study. Sections 5 and 6 are the heart of the paper: Item 1 above is shown in Section 5 through characterization theorems of the extensions of the frameworks and, as a consequence, of the induced entailment relations. We also provide in that section some results on the relations of argumentative entailments and reasoning with consistent subsets of premises (Rescher and Manor 1970), as well as relation to the default assumptions in (Makinson 2003) and adaptive logics (Batens 2007; Straßer 2014), extending known results on the subject. 
Items 2 and 3 are shown through some classification results, in Section 6, that relate argumentative entailments and general patterns for nonmonotonic reasoning (e.g., those in (Kraus, Lehmann, and Magidor 1990) and in (Makinson 1994)), and study inconsistency maintenance (da Costa 1974; Caminada, Carnielli, and Dunne 2011). Finally, in Section 7 we discuss our results and conclude.

\section{Literature Review}

In the context of formal argumentation, postulate-based investigations of argumentation frameworks play a primary role, allowing not only to indicate how the ingredients of the frameworks affect their properties, but also to compare related approaches to argumentation-based reasoning. For instance, studies on the properties of attack relations in logical argumentation appear in (Amgoud and Besnard 2010; Gorogiannis and Hunter 2011; Vesic 2013; Arieli, Borg, and Straßer 2020). Unlike our case, the discussions in these papers (except the last one) are concentrated on classical logic as the base logic of the frameworks, where the supports of the arguments are assumed to be classically consistent and the minimal ones that entail the argument's conclusion (see also Note 1 below). Several properties of extensions of logical argumentation frameworks are studied in, e.g., (Caminada and Amgoud 2007; Amgoud and Besnard 2010; Gorogiannis and Hunter 2011; Amgoud 2014; Arieli, Borg, and Straßer 2020), again (except the last one) with respect to restricted supports of arguments.

This work provides a broad postulate-based overview, including generalizations of existing results as well as new results on the inferential capabilities of argumentative entailments. The interplay between logical principles concerning argumentation, on the one hand, and inference principles as studied in proof theory, on the other hand, is also studied in (Corsi and Fermüller 2017). In that paper a series of logical principles of attack relations in argumentation frameworks is stated, and their collection leads to a characterization of classical logical consequence relations that only involves argumentation frameworks. We refer to (Corsi and Fermüller 2017) and (Corsi and Fermüller 2019) for further details.

Studies of inferential behavior of logical argumentation, and in particular its relation to nonmonotonic reasoning, can also be found in (Arieli and Straßer 2019, Section 5), in the context of dynamic proof systems. Similar studies for ABA and ASPIC systems appear, respectively, in (Čyras and Toni 2015; Heyninck and Arieli 2020; Heyninck and Straßer 2021) and in (Li, Oren, and Parsons 2018).

An important aspect of argumentative inferences, also considered and generalized in this paper, is their relation to reasoning with maximally consistent subsets (Rescher and Manor 1970). Postulates on this kind of reasoning have a primary role in several works and may be traced back to Cayrol (1995). For detailed discussions and surveys on this subject we refer to (Arieli, Borg, and Straßer 2018; Arieli, Borg, and Heyninck 2019). Rationality postulates for other forms of structured argumentation, such as $\mathrm{ASPIC}^{+}$ and ABA systems, can be found, e.g., in (Modgil and Prakken 2013; Modgil and Prakken 2018) (for $\mathrm{ASPIC}^{+}$ systems) and (Čyras and Toni 2016; Heyninck and Straßer 2021) (for ABA systems).

Our study involves some ideas and notions from proof theory. ${ }^{1}$ The main contribution of this work in relation to related works such as the ones mentioned above is that it provides a comprehensive presentation of the semantic as well as the inferential properties of logical argumentation frameworks, where only minimal (proof-theoretic) requirements are made on the base logic and very little is assumed on the form of the arguments. This allows to capture a wide range of core logics and to base arguments only on deducibility in the core logic. In our study, we avoid the use of further conditions (such as conflict dependence and conflict sensitivity that are considered in (Amgoud and Besnard 2010) and (Vesic 2013)) that are computationally demanding, and so are rather difficult to verify.

\section{Logical Argumentation Frameworks}

In the sequel, we denote by $\mathcal{L}$ an arbitrary propositional language. Sets of formulas are denoted by $\mathcal{S}, \mathcal{T}$, finite sets of formulas are denoted by $\Gamma, \Delta$, formulas are denoted by $\phi, \psi$, and atomic formulas are denoted by $p, q, r$, all of which can be primed or indexed. The set of atomic formulas of $\mathcal{L}$ (respectively, the atomic formulas appearing in the formulas of $\mathcal{S}$ ) is denoted $\operatorname{Atoms}(\mathcal{L})$ (respectively, $\operatorname{Atoms}(\mathcal{S})$ ). The set of the (well-formed) formulas of $\mathcal{L}$ is denoted $\operatorname{WFF}(\mathcal{L})$, the power set of $\operatorname{WFF}(\mathcal{L})$ is denoted $\wp(\operatorname{WFF}(\mathcal{L}))$.

Definition 1 (logic). A logic $\mathrm{L}=\langle\mathcal{L}, \vdash\rangle$ consists of a language $\mathcal{L}$ and a consequence relation $\vdash$ on $\wp(\operatorname{WFF}(\mathcal{L})) \times$ $\operatorname{WFF}(\mathcal{L})$, satisfying: reflexivity $(\mathcal{S} \vdash \phi$ if $\phi \in \mathcal{S})$, monotonicity (if $\mathcal{S}^{\prime} \vdash \phi$ and $\mathcal{S}^{\prime} \subseteq \mathcal{S}$, then $\mathcal{S} \vdash \phi$ ), and transitivity (if $\mathcal{S} \vdash \phi$ and $\mathcal{S}^{\prime}, \phi \vdash \psi$ then $\mathcal{S}, \mathcal{S}^{\prime} \vdash \psi$ ).

A logic $\mathrm{L}$ is assumed to be non-trivial (that is, $\mathcal{S} \nvdash \phi$ for some $\mathcal{S} \neq \emptyset$ and $\phi$ ), structural (if $\mathcal{S} \vdash \phi$ then $\{\theta(\psi) \mid \psi \in$ $\mathcal{S}\} \vdash \theta(\phi)$ for every substitution $\theta$ ), and compact (if $\mathcal{S} \vdash \phi$ then $\Gamma \vdash \phi$ for some finite $\Gamma \subseteq \mathcal{S}$ ).

We shall assume that $\mathcal{L}$ contains at least a $\vdash$-negation operator $\neg$, satisfying $p \forall \neg p$ and $\neg p \forall p$ (for atomic $p$ ), and a $\vdash$-conjunction operator $\wedge$, for which $\mathcal{S} \vdash \psi \wedge \phi$ iff $\mathcal{S} \vdash \psi$ and $\mathcal{S} \vdash \phi$. Also, we denote by $\wedge \Gamma$ the conjunction of all the formulas in (the finite set) $\Gamma$.

Let $\mathrm{L}=\langle\mathcal{L}, \vdash\rangle$ be a logic and let $\mathcal{S}$ be a set of $\mathcal{L}$-formulas. The $\vdash$-closure of $\mathcal{S}$ is the set $\mathrm{CN}_{\mathrm{L}}(\mathcal{S})=\{\phi \mid \mathcal{S} \vdash \phi\}$. We say that $\mathcal{S}$ is $\vdash$-consistent, if there are no formulas $\phi_{1}, \ldots, \phi_{n} \in \mathcal{S}$ for which $\vdash \neg\left(\phi_{1} \wedge \cdots \wedge \phi_{n}\right)$.

Given a logic $\mathrm{L}=\langle\mathcal{L}, \vdash\rangle$, an $\mathcal{L}$-sequent (a sequent for short) (Gentzen 1934) is an expression of the form $\Gamma \Rightarrow \Delta$, where $\Rightarrow$ is a symbol that does not appear in $\mathcal{L}$. An Largument is then a single-conclusion sequent, representing $\mathcal{L}$-entailments:

Definition 2 (argument). Let $L=\langle\mathcal{L}, \vdash\rangle$ be a logic and let $\mathcal{S}$ be a set of formulas in $\mathcal{L}$. An L-argument (argu-

\footnotetext{
${ }^{1}$ The incorporation of proof theoretical concepts and techniques in order to investigate and implement specific logical argumentation frameworks is not new. Cf. (Dunne and Bench-Capon 2003; Grossi 2009; Straßer and Šešelja 2010; Grossi 2011; Arieli and Straßer 2016; Borg and Straßer 2018; Arieli and Straßer 2019).
} 
ment for short) is an $\mathcal{L}$-sequent of the form $\Gamma \Rightarrow \psi,{ }^{2}$ where $\Gamma \vdash \psi$. We say that $\Gamma$ is the support set of $\Gamma \Rightarrow \psi$ (denoted $\operatorname{Supp}(\Gamma \Rightarrow \psi))$ and that $\psi$ is its conclusion (denoted $\operatorname{Conc}(\Gamma \Rightarrow \psi))$. For a set $S$ of arguments, we let $\operatorname{Supps}(\mathrm{S})=$ $\bigcup\{\operatorname{Supp}(a) \mid a \in \mathrm{S}\}$ and $\operatorname{Concs}(\mathrm{S})=\{\operatorname{Conc}(a) \mid a \in \mathrm{S}\}$. An L-argument based on $\mathcal{S}$ is an $\mathrm{L}$-argument $\Gamma \Rightarrow \psi$, where $\Gamma \subseteq \mathcal{S}$. We denote by $\operatorname{Arg}_{\mathrm{L}}(\mathcal{S})$ the set of all the $\mathrm{L}$-arguments based on $\mathcal{S}$.

Note 1. It is sometimes assumed that the argument's support is $\vdash$-consistent and/or $\subseteq$-minimal, that is: none of its proper subsets $\vdash$-entails the arguments' conclusion (see, e.g., (Besnard and Hunter 2009; Amgoud and Besnard 2013)). To keep the presentation as general as possible, we do not make such restrictions. See also the discussions in (Arieli and Straßer 2015; Arieli and Straßer 2020; D'Agostino and Modgil 2020).

In what follows we shall assume that the logic $L=\langle\mathcal{L}, \vdash\rangle$ is equipped with a sound and complete sequent calculus $\mathrm{C}$ for constructing sequents (and so arguments). A sequent is provable (or derivable) in $\mathrm{C}$ if there is a derivation for it in C. Thus, $\Gamma \Rightarrow \psi$ is provable in $\mathrm{C}$ iff $\Gamma \vdash \psi$. Note that this implies, in particular, that for a given set $\mathcal{S}$, all the elements in $\operatorname{Arg}_{\mathrm{L}}(\mathcal{S})$ are C-provable.

In structured argumentation it is often distinguished between two types of premises: strict (i.e., non-attacked) and defeasible ones (Dung, Kowalski, and Toni 2009; Modgil and Prakken 2013; Borg 2020). To accommodate this difference in our setting we consider a $\vdash$-consistent set of $\mathcal{L}$ formulas $\mathcal{X}$ as strict premises. Their non-defeasible character will give them a special status when we define argumentative attacks below. To distinguish between $\mathcal{X}$ and the set $\mathcal{S}$ of the other premises, we write $\operatorname{Arg}_{\mathrm{L}}^{\mathcal{X}}(\mathcal{S})$ for the set $\operatorname{Arg}_{\mathrm{L}}(\mathcal{X} \cup \mathcal{S})$. In particular, $\operatorname{Arg}_{\mathrm{L}}^{\emptyset}(\mathcal{S})=\operatorname{Arg}_{\mathrm{L}}(\mathcal{S})$.

Just as arguments are constructed by inference rules in $C$, attacks between arguments can be represented by (attack) rules. Such rules consist of an attacking argument (the first condition of the rule), an attacked argument (the last condition of the rule), conditions for the attack (the other conditions of the rule) and a conclusion (the eliminated attacked sequent). The outcome of an application of such a rule is that the attacked sequent is 'eliminated' (or 'invalidated'; see below the exact meaning of this). The elimination of a sequent $\Gamma \Rightarrow \phi$ is denoted by $\Gamma \nRightarrow \phi$.

Definition 3 (attack rules). Given a set $\mathcal{X}$ of strict (nonattacked) formulas, we consider the following attack rules: ${ }^{3}$

- Defeat $\left(\operatorname{Def}_{\mathcal{X}}\right)$ : for $\Gamma_{2} \neq \emptyset, \Gamma_{2} \cap \mathcal{X}=\emptyset$,

$$
\frac{\Gamma_{1} \Rightarrow \psi_{1} \quad \psi_{1} \Rightarrow \neg \bigwedge \Gamma_{2} \quad \Gamma_{2}, \Gamma_{2}^{\prime} \Rightarrow \psi_{2}}{\Gamma_{2}, \Gamma_{2}^{\prime} \nRightarrow \psi_{2}}
$$

- Direct Defeat $\left(\operatorname{DDef}_{\mathcal{X}}\right)$ : for $\gamma \notin \mathcal{X}$,

\footnotetext{
${ }^{2}$ Set signs in arguments are omitted.

${ }^{3}$ For $\mathcal{X}=\emptyset$ these are exactly the rules from (Arieli and Straßer 2015; Straßer and Arieli 2019). There, the reader can also find many other rules. In (Borg 2020) sequent-based argumentation has been generalized along similar lines, where the left side of a sequent is a pair $\Pi \mid \Gamma$ consisting of a set of defeasible premises $\Pi$ and a set of strict premises $\Gamma$.
}

$$
\frac{\Gamma_{1} \Rightarrow \psi_{1} \quad \psi_{1} \Rightarrow \neg \gamma \quad \Gamma_{2}, \gamma \Rightarrow \psi_{2}}{\Gamma_{2}, \gamma \neq \nRightarrow \psi_{2}}
$$

- Undercut (Ucut $\mathcal{X})$ : for $\Gamma_{2} \neq \emptyset, \Gamma_{2} \cap \mathcal{X}=\emptyset$,

$$
\frac{\Gamma_{1} \Rightarrow \psi_{1} \quad \psi_{1} \Rightarrow \neg \bigwedge \Gamma_{2} \quad \neg \bigwedge \Gamma_{2} \Rightarrow \psi_{1} \quad \Gamma_{2}, \Gamma_{2}^{\prime} \Rightarrow \psi_{2}}{\Gamma_{2}, \Gamma_{2}^{\prime} \not \psi_{2}}
$$

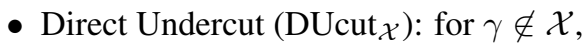

$$
\frac{\Gamma_{1} \Rightarrow \psi_{1} \quad \psi_{1} \Rightarrow \neg \gamma \quad \neg \gamma \Rightarrow \psi_{1} \quad \Gamma_{2}, \gamma \Rightarrow \psi_{2}}{\Gamma_{2}, \gamma \nRightarrow \nRightarrow \psi_{2}}
$$

- Cons. Ucut (ConUcut $\mathcal{X}$ ): for $\Gamma_{2} \neq \emptyset, \Gamma_{2} \cap \mathcal{X}=\emptyset, \Gamma_{1} \subseteq \mathcal{X}$,

$$
\frac{\Gamma_{1} \Rightarrow \neg \bigwedge \Gamma_{2} \quad \Gamma_{2}, \Gamma_{2}^{\prime} \Rightarrow \psi}{\Gamma_{2}, \Gamma_{2}^{\prime} \nRightarrow \psi}
$$

The rules above indicate when the attacker challenges the attacked argument. For instance, when $\{p, \neg p\} \subseteq \mathcal{S}, \mathcal{X}=\emptyset$ and classical logic (CL) is the core logic, the sequents $p \Rightarrow p$ and $\neg p \Rightarrow \neg p$ attack each other according to (Direct) Defeat and (Direct) Undercut. When $\mathcal{S}=\{\neg p\}$ and $\mathcal{X}=\{p\}$ the attack is uni-directional from $p \Rightarrow p$ to $\neg p \Rightarrow \neg p$, since an argument cannot be attacked in its strict premises.

An argumentation framework is now defined as follows:

Definition 4 (argumentation framework). A (sequentbased) argumentation framework (AF), based on the logic $L$ and the attack rules in $A$, for a set of defeasible premises $\mathcal{S}$ and a $\vdash$-consistent set of strict premises $\mathcal{X}$, is a pair $\mathcal{A} \mathcal{F}_{\mathrm{L}, \mathrm{A}}^{\mathcal{X}}(\mathcal{S})=\left\langle\operatorname{Arg}_{\mathrm{L}}^{\mathcal{X}}(\mathcal{S}), \mathcal{A}\right\rangle$ where $\mathcal{A} \subseteq \operatorname{Arg}_{\mathrm{L}}^{\mathcal{X}}(\mathcal{S}) \times \operatorname{Arg}_{\mathrm{L}}^{\mathcal{X}}(\mathcal{S})$ and $\left(a_{1}, a_{2}\right) \in \mathcal{A}$ iff there is a rule $\mathcal{R}_{\mathcal{X}} \in \mathrm{A}$ such that $a_{1}$

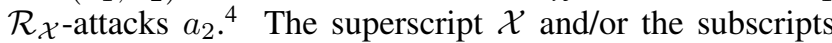
$\mathrm{L}, \mathrm{A}$ will be omitted when they are clear from the context or arbitrary.

Example 1. Consider the modal logic S4 and the let $\mathcal{S}=$ $\{q, p \supset \square r, q \supset \square \neg r\}$ and $\mathcal{X}=\{p\}$. Some arguments in $\operatorname{Arg}_{S 4}(\mathcal{S})$ are the following:

$$
\begin{array}{ll}
a_{1}: p \Rightarrow p & a_{2}: q \Rightarrow q \\
a_{3}: p, p \supset \square r \Rightarrow \square r & a_{4}: q, q \supset \square \neg r \Rightarrow \square \neg r \\
a_{5}: p, p \supset \square r, q \supset \square \neg r \Rightarrow \neg q & a_{6}: p, q, p \supset \square r \Rightarrow \neg(q \supset \square \neg r) \\
a_{7}: p, q, q \supset \square \neg r \Rightarrow \neg(p \supset \square r) & a_{8}: q, p \supset \square r, q \supset \square \neg r \Rightarrow \neg p
\end{array}
$$

Figure 1 depicts (part of) the argumentation framework for this setting, with direct defeat as the sole attack rule. In the figure, an arrow from $a$ to $b$ means that $a$ attacks $b$.

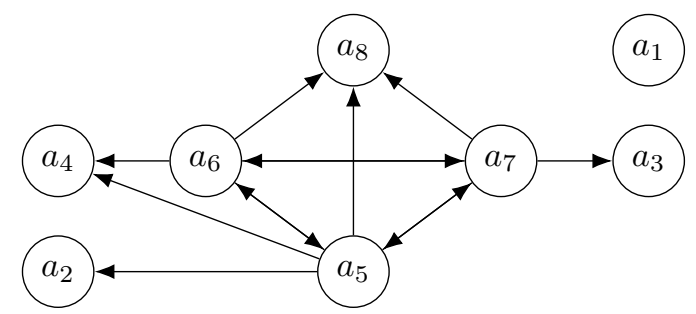

Figure 1: A representation of the framework of Example 1.

\footnotetext{
${ }^{4}$ The attacking and the attacked arguments must be elements of $\operatorname{Arg}_{\mathrm{L}}^{\mathcal{X}}(\mathcal{S})$, to prevent "irrelevant attacks", in which, e.g., $\neg p \Rightarrow \neg p$ attacks $p \Rightarrow p$ although $\mathcal{S}=\{p\}$.
} 
Given an argumentation framework $\mathcal{A} \mathcal{F}_{\mathrm{L}, \mathrm{A}}^{\mathcal{X}}(\mathcal{S})$, Dungstyle semantics (Dung 1995) can be applied to it, to determine what combinations of arguments (called extensions) can collectively be accepted from $\mathcal{A} \mathcal{F}_{\mathrm{L}, \mathrm{A}}^{\mathcal{X}}(\mathcal{S})$.

Definition 5 (extensions). Let $\mathcal{A F}=\mathcal{A F}_{\mathrm{L}, \mathrm{A}}^{\mathcal{X}}(\mathcal{S})=$ $\left\langle\operatorname{Arg}_{\mathrm{L}}^{\mathcal{X}}(\mathcal{S}), \mathcal{A}\right\rangle$ be an argumentation framework and let $\mathrm{S} \subseteq$ $\operatorname{Arg}_{\mathrm{L}}^{\mathcal{X}}(\mathcal{S})$ be a set of arguments. It is said that:

- S attacks $a$ if there is an $a^{\prime} \in \mathrm{S}$ such that $\left(a^{\prime}, a\right) \in \mathcal{A}$. The set of all arguments attacked by $S$ is denoted by $S^{+}$.

- S defends $a$ if $\mathrm{S}$ attacks every attacker of $a$.

- $\mathrm{S}$ is conflict-free (cf) if for no $a_{1}, a_{2} \in \mathrm{S},\left(a_{1}, a_{2}\right) \in \mathcal{A}$.

- $\mathrm{S}$ is a stage (stg) extension of $\mathcal{A F}$ if $\mathrm{S} \cup \mathrm{S}^{+}$is $\subseteq$-maximal among the conflict-free sets.

- $\mathrm{S}$ is admissible (adm) if it is conflict-free and defends all of its elements.

- A complete (cmp) extension of $\mathcal{A F}$ is an admissible set that contains all the arguments that it defends.

- The grounded (grd) extension of $\mathcal{A F}$ is the $\subseteq$-minimal complete extension of $\operatorname{Arg}_{\mathrm{L}}^{\mathcal{X}}(\mathcal{S})$.

- A preferred (prf) extension of $\mathcal{A F}$ is a $\subseteq$-maximal complete extension of $\operatorname{Arg}_{\mathrm{L}}^{\mathcal{X}}(\mathcal{S})$.

- The ideal (idl) extension of $\mathcal{A F}$ is the $\subseteq$-maximal admissible set that is included in each preferred extension.

- A stable (stb) extension of $\mathcal{A F}$ is a conflict-free set in $\operatorname{Arg}_{\mathrm{L}}^{\mathcal{X}}(\mathcal{S})$ that attacks every argument not in it.

- A semi-stable (sstb) extension $\mathrm{S}$ of $\mathcal{A F}$ is a complete extension for which $S \cup \mathrm{S}^{+}$is $\subseteq$-maximal.

- The eager (egr) extension of $\mathcal{A F}$ is the $\subseteq$-maximal admissible set included in every semi-stable extension.

We denote by $\operatorname{Ext}_{\text {sem }}(\mathcal{A F})$ the set of all the extensions of $\mathcal{A} \mathcal{F}$ of type sem for some sem $\in\{\mathrm{stg}, \mathrm{cmp}$, grd, prf, idl, stb, sstb, egr $\}$. The subscript is omitted when it is clear from the context.

We are now ready to define the entailment relations that are induced from a given sequent-based argumentation framework and its semantics.

Definition 6 (entailments). Given an argumentation framework $\mathcal{A F}=\mathcal{A} \mathcal{F}_{\mathrm{L}, \mathrm{A}}^{\mathcal{X}}(\mathcal{S})$ and a semantics sem for it, the following entailment relations are induced from $\mathcal{A F}$ :

- Skeptical entailment: $\mathcal{S} \sim_{\mathrm{L}, \mathrm{A}, \mathcal{X}}^{\cap, \mathrm{X}} \phi$ if there is an argument $a \in \bigcap \operatorname{Ext}_{\text {sem }}(\mathcal{A F})$ such that $\operatorname{Conc}(a)=\phi$.

- Weakly skeptical entailment: $\mathcal{S} \sim_{\text {L,A, }}^{\text {ก,sem }} \phi$ if for every extension $\mathcal{E} \in \operatorname{Ext}_{\text {sem }}(\mathcal{A F})$ there is an argument $a \in \mathcal{E}$ such that $\operatorname{Conc}(a)=\phi$.

- Credulous entailment: $\mathcal{S} \sim_{\mathrm{L}, \mathrm{A}, \mathcal{X}}^{\cup, s e m} \phi$ iff there is an argument $a \in \bigcup \operatorname{Ext}_{\text {sem }}(\mathcal{A F})$ such that $\operatorname{Conc}(a)=\phi$.

For fixed L, A and sem, it holds that $\sim_{\mathrm{L}, \mathrm{A}, \mathcal{X}}^{\cap, \mathrm{sem}} \subseteq \sim_{\mathrm{L}, \mathrm{A}, \mathcal{X}}^{\mathrm{n}, \text { sem }} \subseteq$ $\sim_{\mathrm{L}, \mathrm{A}, \mathcal{X}}^{\cup}$. The superscript $\mathcal{X}$ and the subscripts $\mathrm{L}, \mathrm{A}$, sem are omitted in this and other notations when they are clear from the context or arbitrary. Since the grounded, ideal and eager extensions are unique (see, e.g., (Baroni, Caminada, and Giacomin 2018)), $\sim_{\mathrm{L}, \mathrm{A}, \mathcal{X}}^{\cap, \mathrm{X}}, \sim_{\mathrm{L}, \mathrm{A}, \mathcal{X}}^{\mathrm{M}}$ and $\sim_{\mathrm{L}, \mathrm{A}, \mathcal{X}}$,sem coincide for sem $\in\{$ grd, idl, egr $\}$, they are sometimes denoted $\sim_{\mathrm{L}, A, \mathcal{X}}^{\text {sem }}$.
Example 2. Consider again the framework of Example 1 and Figure 1. The preferred extensions in this case are:

$$
\begin{aligned}
& \operatorname{Arg}_{\mathrm{S} 4}^{\{p\}}(\{q, p \supset \square r\}), \quad \operatorname{Arg}_{\mathrm{S} 4}^{\{p\}}(\{q, q \supset \square \neg r\}), \\
& \operatorname{Arg}_{\mathrm{S} 4}^{\{p\}}(\{p \supset \square r, q \supset \square \neg r\}) .
\end{aligned}
$$

These are also the (semi-)stable extensions of the framework. Thus, although $\mathcal{S} \cup \mathcal{X}$ is inconsistent with respect to $\vdash_{\mathrm{s} 4}$, argumentative reasoning is not degenerated in this case. For instance, it holds that $\mathcal{S} \mathcal{W}_{\mathrm{S} 4,\{\text { DDef }\},\{p\}}^{\star, \text { em }} \psi$ for any $\psi \in \mathcal{S}, \star \in\{\cap, \cap\}$, and sem $\in\{$ prf, stb, sstb $\}$, but we do have that, e.g., $\mathcal{S} \sim_{\mathrm{S} 4,\{\text { DDef }\},\{p\}}^{\star \text { sem }}(p \supset \square r) \vee(q \supset \square \neg r)$ when $\star \in\{\cap, \cup\}$ and sem $\in\{$ prf, stb, sstb $\}$.

\section{The Scope of Our Study}

Despite the diversity of the logics and their sequent calculi covered in this work, for our results not too many assumptions will be made about the actual content of the calculi. In fact, we only need to assume that the rules of the basic calculus from Figure 2 are part of (or admissible in) C.

$$
\begin{aligned}
& \text { [Ref] } \frac{\overline{\phi \Rightarrow \phi}}{{ }^{\text {Cut }]} \frac{\Gamma_{1} \Rightarrow \psi, \Pi_{1} \quad \Gamma_{2}, \psi \Rightarrow \Delta_{2}}{\Gamma_{1}, \Gamma_{2} \Rightarrow \Pi_{1}, \Delta_{2}}} \\
& \text { [LMon] } \frac{\Gamma \Rightarrow \Delta}{\Gamma, \phi \Rightarrow \Delta} \quad[\text { RMon }] \frac{\Gamma \Rightarrow \Pi}{\Gamma \Rightarrow \Pi, \phi} \\
& {[\neg \Rightarrow] \frac{\Gamma \Rightarrow \Pi, \varphi}{\neg \varphi, \Gamma \Rightarrow \Pi} \quad[\Rightarrow \neg] \frac{\varphi, \Gamma \Rightarrow \Pi}{\Gamma \Rightarrow \Pi, \neg \varphi}} \\
& {[\wedge \Rightarrow] \frac{\Gamma, \varphi, \psi \Rightarrow \Delta}{\Gamma, \varphi \wedge \psi \Rightarrow \Delta} \quad[\Rightarrow \wedge] \frac{\Gamma_{1} \Rightarrow \Pi_{1}, \varphi \quad \Gamma_{2} \Rightarrow \Pi_{2}, \psi}{\Gamma_{1}, \Gamma_{2} \Rightarrow \Pi_{1}, \Pi_{2}, \varphi \wedge \psi}}
\end{aligned}
$$

Figure 2: Rules that are part of (or admissible in) the calculus $C$ (in the case that $C$ is single-conclusioned $\Pi, \Pi_{1}$ and $\Pi_{2}$ should be empty and $\Delta$ and $\Delta_{2}$ contain at most one formula).

The first four rules correspond to the properties of consequence relations (Definition 1): reflexivity [Ref], transitivity [Cut] and monotonicity [LMon, RMon]; The other four rules refer to the behavior of negation in the left-hand side of sequents $[\neg \Rightarrow]$, in the right-hand side $[\Rightarrow \neg]$, and similar rules for conjunction $([\wedge \Rightarrow]$ and $[\Rightarrow \wedge]$, respectively).

Example 3. Gentzen's sequent calculus LK for classical logic, its single-conclusion variation $\mathrm{LJ}$ for intuitionistic logic, as well as their extensions to modal logics (including the base logic S4 in Examples 1 and 2), are some wellknown calculi for base logics that are covered by our study.

In what follows we shall examine three types of attacks in argumentation frameworks: set, dir and con.

Definition 7 (types of attacks). A set $A$ of of attack rules may be of one of the following types:

set: attack rules in which an argument is attacked on a

(sub)set of its support, and where at least one of the rules is Undercut or Defeat (i.e., $A \cap\left\{\operatorname{Def}_{\mathcal{X}}, \operatorname{Ucut}_{\mathcal{X}}\right\} \neq \emptyset$ ),

dir: nonempty sets of direct attack rules (that is, $\emptyset \neq A \subseteq$ $\left\{\right.$ DDef $\left.\left._{\mathcal{X}}, \operatorname{DUcut}_{\mathcal{X}}\right\}\right)$ 
con: dir-type set of rules that also contain ConUcut (that is, $\left\{\right.$ ConUcut $\left._{\mathcal{X}}\right\} \subsetneq \mathrm{A} \subseteq\left\{\right.$ ConUcut $_{\mathcal{X}}$, DDef $_{\mathcal{X}}$, DUcut $\left.\left._{\mathcal{X}}\right\}\right)$.

Note 2. Any set of attacks from Definition 3 for which $\mathrm{A} \backslash\{$ ConUcut $\} \neq \emptyset$ falls in one of the three types above. Moreover, these types are disjoint.

Our results will apply to completeness-based semantics.

Definition 8 (completeness-based semantics). A semantics sem (e.g., one of those in Definition 5) is completenessbased, iff for every argumentation framework $\mathcal{A F}$ it holds that $\operatorname{Ext}_{\mathrm{sem}}(\mathcal{A F}) \subseteq \operatorname{Ext}_{\mathrm{cmp}}(\mathcal{A F})$.

Note 3. By their definitions, complete, grounded, and preferred semantics are all completeness-based relative to any type of attacks. Since it can be shown that every stable, semi-stable, ideal, and eager extension is always complete (see, e.g., (Baroni, Caminada, and Giacomin 2018)), the same holds for these semantics. In general, stage extensions need not be complete. However, the following result shows that for the three types of attacks in Definition 7, also stage semantics is completeness-based. Therefore, in this paper, we will call stage semantics completeness-based as well.

Proposition 1. If $\mathcal{A F}$ is a framework with attacks of one of the types in Definition 7, then $\operatorname{Ext}_{\mathrm{stb}}(\mathcal{A F})=\operatorname{Ext}_{\mathrm{stg}}(\mathcal{A F})$.

Proof outline. The direction $\operatorname{Ext}_{\mathrm{stb}}(\mathcal{A F}) \subseteq \operatorname{Ext}_{\mathrm{stg}}(\mathcal{A F})$ is trivial. For the converse, we note that any framework with attacks of a type in Definition 7 has a stable extension. ${ }^{5}$ Suppose then that $\mathcal{E} \in \operatorname{Ext}_{\text {stg }}(\mathcal{A F})$ and let $\mathcal{E}^{\prime} \in \operatorname{Ext}_{\text {stb }}(\mathcal{A F})$. Since $\mathcal{E}^{\prime}$ is conflict-free and $\mathcal{E}^{\prime} \cup \mathcal{E}^{+}=\operatorname{Arg}(\mathcal{A F})$, and since $\mathcal{E}^{\prime} \cup \mathcal{E}^{\prime+}$ is $\subseteq$-maximal among the conflict-free sets, also $\mathcal{E} \cup \mathcal{E}^{+}=\operatorname{Arg}(\mathcal{A F})$. Thus, $\mathcal{E} \in \operatorname{Ext}_{\text {stb }}(\mathcal{A F})$.

Notation 1. We denote by CMP the set of completenessbased semantics, and by ME (respectively, by SE) the subset of multiple-extension (respectively, single-extension) semantics, namely: ${ }^{6}$

- $\mathrm{CMP}=\{\mathrm{cmp}$, prf, stb, sstb, stg, grd, idl, egr $\}$,

- $M E=\{$ prf, stb, sstb, stg $\}$,

- $\mathrm{SE}=\{$ grd, idl, egr $\}$.

In what follows we then consider argumentation frameworks based on any propositional logic $L$ with a sound and complete sequent calculus $\mathrm{C}$, in which the rules in Figure 2 are admissible, any set of assumptions (strict and/or deafeasible) in the language of the logic, any set of attack rules A of any one of the three types given in Definition 7, and any semantics sem in CMP. To the best of our knowledge, this variety has not been considered previously in the literature.

\section{Characterizations of Extensions and Entailment Relations}

In this section we characterize the extensions in Definition 5 and the entailment relations in Definition 6, induced by argumentation frameworks with attacks of the types described in Definition 7. For this, we need the following notations of different types of consistent sets of formulas.

\footnotetext{
${ }^{5}$ E.g., by Theorem 2 and the existence of preferred extensions.

${ }^{6}$ The exclusion of $\mathrm{cmp}$ from ME is explained in Note 4.
}

Notation 2. For a logic $\mathrm{L}$, a set $\mathcal{S}$ of formulas and a $\vdash$ consistent set $\mathcal{X}$ of formulas, we say that $\mathcal{S}$ is $\vdash \mathcal{X}$-consistent if $\mathcal{S} \cup \mathcal{X}$ is $\vdash$-consistent. We denote.

- $\operatorname{CS}_{\mathrm{L}}^{\mathcal{X}}(\mathcal{S})$ is the set of the $\vdash_{\mathcal{X}}$-consistent subsets of $\mathcal{S}$, and $\operatorname{MCS}_{\mathrm{L}}^{\mathcal{X}}(\mathcal{S})$ consists of the $\subseteq$-maximal elements in $\mathrm{CS}_{\mathrm{L}}^{\mathcal{X}}(\mathcal{S})$.

- $\Omega_{\mathrm{L}}^{\mathcal{X}}(\mathcal{S})=\{\omega \mid \omega \subseteq \wp(\mathcal{S})\}$ is the set of subsets of $\wp(\mathcal{S})$, satisfying the following two requirements:

a) the elements of $\omega$ are pairwise $\vdash \mathcal{X}$-consistent: $\mathcal{T}_{i} \cup \mathcal{T}_{j}$ is $\vdash_{\mathcal{X}}$-consistent for every $\mathcal{T}_{i}, \mathcal{T}_{j} \in \omega$.

b) for every finite $\mathcal{T} \in \wp(\mathcal{S})$ there is a $\mathcal{T}^{\prime} \in \omega$ such that either $\mathcal{T} \subseteq \mathcal{T}^{\prime}$ or $\mathcal{T} \cup \mathcal{T}^{\prime}$ is $\vdash$ - -inconsistent.

For $\omega \in \Omega_{\mathrm{L}}^{\mathcal{X}}(\mathcal{S})$, we let $\operatorname{Arg}_{\mathrm{L}}^{\mathcal{X}}(\omega)=\bigcup_{\mathcal{T} \in \omega} \operatorname{Arg}_{\mathrm{L}}^{\mathcal{X}}(\mathcal{T})$.

We are now ready for the first characterization result. For $\mathcal{X}=\emptyset$, the first item in the following theorem, for preferred and stable semantics, is known from the literature (see (Arieli, Borg, and Straßer 2020)); the other items are new.

Theorem 1. Given an argumentation framework $\mathcal{A F}_{\mathrm{L}, \mathrm{A}}^{\mathcal{X}}(\mathcal{S})$ based on a logic $\mathrm{L}$, a set $\mathrm{A}$ of attack rules that are of type $\dagger \in\{\operatorname{dir}$, con, set $\}$. It holds that:

1. $\operatorname{Ext}_{\text {sem }}\left(\mathcal{A F}_{\mathrm{L}, \mathrm{A}}^{\mathcal{X}}(\mathcal{S})\right)=\left\{\operatorname{Arg}_{\mathrm{L}}^{\mathcal{X}}(\mathcal{T}) \mid \mathcal{T} \in \operatorname{MCS}_{\mathrm{L}}^{\mathcal{X}}(\mathcal{S})\right\}$ when $\dagger \in\{$ dir, con $\}$ and sem $\in$ ME.

2. $\operatorname{Ext}_{\text {sem }}\left(\mathcal{A F}_{\mathrm{L}, \mathrm{A}}^{\mathcal{X}}(\mathcal{S})\right)=\left\{\operatorname{Arg}_{\mathrm{L}}^{\mathcal{X}}(\omega) \mid \omega \in \Omega_{\mathrm{L}}^{\mathcal{X}}(\mathcal{S})\right\}$ when $\dagger=$ set and sem $\in \mathrm{ME}$.

3. $\operatorname{Ext}_{\text {sem }}\left(\mathcal{A F}_{\mathrm{L}, \mathrm{A}}^{\mathcal{X}}(\mathcal{S})\right)=\left\{\operatorname{Arg}_{\mathrm{L}}^{\mathcal{X}}\left(\operatorname{Free}_{\mathrm{L}}^{\mathcal{X}}(\mathcal{S})\right)\right\}$ when $\dagger \in\{$ con, set $\}$ and sem $\in \mathrm{SE}$, and where $\operatorname{Free}_{\mathrm{L}}^{\mathcal{X}}(\mathcal{S})$ is the set of formulas from $\mathcal{S}$ that are not part of any $\subseteq$ minimal $\vdash \mathcal{X}$-inconsistent subset of $\mathcal{S}$.

4. $\operatorname{Ext}_{\text {sem }}\left(\mathcal{A} \mathcal{F}_{\mathrm{L}, \mathrm{A}}^{\mathcal{X}}(\mathcal{S})\right)=\left\{\operatorname{Arg}_{\mathrm{L}}^{\mathcal{X}}(\mathcal{T})\right\}$ when $\dagger=$ dir and sem $\in \mathrm{SE}$, and where $\mathcal{S}_{\mathrm{T}}=\{\phi \in$ $\mathcal{S} \mid \phi$ is $\vdash_{\mathcal{X}}$-consistent $\}$ and $\mathcal{T}=\mathcal{S}_{\top}$ in case $\mathcal{S}_{\top}$ is $\vdash_{\mathcal{X}}$ consistent and $\mathcal{T}=\emptyset$ otherwise.

Proof outline. We show here Item 4, leaving the other items to the full version of the paper.

We denote: $\mathcal{S}_{T}=\left\{\phi \in \mathcal{S} \mid \phi\right.$ is $\vdash_{\mathcal{X}}$-consistent $\}$. Let $\operatorname{Ext}_{\text {sem }}\left(\mathcal{A} \mathcal{F}_{\mathrm{L}, \mathrm{A}}^{\mathcal{X}}(\mathcal{S})\right)=\{\mathcal{E}\}$ and $\mathcal{S}_{\perp}=\mathcal{S} \backslash \mathcal{S}_{\top}$. Note that for each $\phi \in \mathcal{S}_{\perp}$, there is a $\Xi \subseteq \mathcal{X}$ such that $\Xi \Rightarrow \neg \phi \in$ $\operatorname{Arg}_{\llcorner}{ }_{\llcorner}^{\mathcal{X}}(\emptyset)$ attacks any argument $a$ with $\phi \in \operatorname{Supp}(a)$. Also, $\operatorname{Arg}_{\mathrm{L}}^{\mathcal{X}}(\emptyset) \subseteq \mathcal{E}$, since these arguments have no attackers.

Suppose first that $\mathcal{S}_{\top}$ is $\vdash_{\mathcal{X}}$-consistent. It can be easily verified that $\operatorname{Arg}_{\mathrm{L}}^{\mathcal{X}}\left(\mathcal{S}_{T}\right)$ is conflict-free. Assume that some $b \in \operatorname{Arg}_{\mathrm{L}}^{\mathcal{X}}(\mathcal{S})$ attacks some $a \in \operatorname{Arg}_{\mathrm{L}}^{\mathcal{X}}\left(\mathcal{S}_{T}\right)$. Thus, $\operatorname{Supp}(b) \cap$ $\mathcal{S}_{\perp} \neq \emptyset$ and hence $b$ is attacked by $\operatorname{Arg}_{\mathrm{L}}^{\mathcal{X}}(\emptyset)$. So, $\operatorname{Arg}_{\mathrm{L}}^{\mathcal{X}}\left(\mathcal{S}_{\mathrm{T}}\right)$ is defended by $\operatorname{Arg}_{\mathrm{L}}{ }^{\mathcal{X}}(\emptyset)$. Since $\operatorname{Arg}_{\mathrm{L}}{ }^{\mathcal{X}}(\emptyset) \subseteq \mathcal{E}$ and by the completeness of $\mathcal{E}, \operatorname{Arg}_{\mathrm{L}}^{\mathcal{X}}\left(\mathcal{S}_{\top}\right) \subseteq \mathcal{E}$. We have already seen that any argument $a^{\prime}$ with $\operatorname{Supp}\left(a^{\prime}\right) \cap \mathcal{S}_{\top} \neq \emptyset$ is attacked by $\mathcal{E}$, and so $\mathcal{E}=\operatorname{Arg}_{\mathrm{L}}^{\mathcal{X}}\left(\mathcal{S}_{\top}\right)$.

Suppose now that $\mathcal{S}_{\top}$ is $\vdash_{\mathcal{X}}$-inconsistent and let $\Gamma$ be a finite $\vdash_{\mathcal{X}}$-inconsistent subset of $\mathcal{S}_{\top}$. Assume for a contradiction that $\operatorname{Arg}_{\mathrm{L}}^{\mathcal{X}}(\emptyset) \subsetneq \mathcal{E}$. Since by the definition of sem, $\mathcal{E}$ is contained in every stable extension and by Item 1 , it follows that $\bigcup\{\operatorname{Supp}(a) \backslash \mathcal{X} \mid a \in \mathcal{E}\} \subseteq \bigcap \operatorname{MCS}_{\mathrm{L}}^{\mathcal{X}}(\mathcal{S})=\operatorname{Free}_{\mathrm{L}}^{\mathcal{X}}(\mathcal{S})$. Let $a \in \mathcal{E}$ with $\operatorname{Supp}(a) \backslash \mathcal{X} \neq \emptyset$ and let $\phi \in \operatorname{Supp}(a) \backslash \mathcal{X}$. 
Thus, for some $\Xi_{b} \subseteq \mathcal{X}, b=\Gamma \cup \Xi_{b} \Rightarrow \neg \phi$ attacks $a$. Hence, there is a $c=\Delta \Rightarrow \psi \in \mathcal{E}$ that attacks $b$. So there is a $\gamma \in \Gamma$ for which $\psi \Rightarrow \neg \gamma$ is derivable, and by [Cut], $\Delta \Rightarrow \neg \gamma$ is also derivable. Hence $(\Delta \cup\{\gamma\}) \backslash \mathcal{X}$ is $\vdash \mathcal{X}-$ inconsistent. Thus there is a minimally $\vdash \mathcal{X}$-inconsistent set $\Theta \subseteq(\Delta \cup\{\gamma\}) \backslash \mathcal{X}$, and since $\Delta \backslash \mathcal{X} \subseteq \operatorname{Free}_{\mathrm{L}}^{\mathcal{X}}(\mathcal{S}), \Theta=\{\gamma\}$, i.e., $\gamma$ is $\vdash \mathcal{X}$-inconsistent. Hence, $\Xi \Rightarrow \neg \gamma$ is derivable for some $\Xi \subseteq \mathcal{X}$, which is a contradiction to $\gamma \in \mathcal{S}_{\top}$. Altogether, this shows that $\mathcal{E}=\operatorname{Arg}_{\mathrm{L}}^{\mathcal{X}}(\emptyset)$.

Theorem 1 implies that we are essentially dealing with only two sub-classes of completeness-based semantics:

Theorem 2. Let $\mathcal{A F}$ be a logical argumentation framework whose set of attacks is of type dir, con or set. Then:

1. $\operatorname{Ext}_{\text {prf }}(\mathcal{A F})=\operatorname{Ext}_{\text {stb }}(\mathcal{A F})=\operatorname{Ext}_{\text {sstb }}(\mathcal{A F})=\operatorname{Ext}_{\text {stg }}(\mathcal{A F})$,

2. $\operatorname{Ext}_{\text {grd }}(\mathcal{A F})=\operatorname{Ext}_{\text {idl }}(\mathcal{A F})=\operatorname{Ext}_{\text {egr }}(\mathcal{A F})$.

Theorem 2 can also be expressed in terms of entailment relations. For this, we shall somewhat modify the notations of the entailments in Definition 6 and sometimes use the abbreviation $\sim_{\mathrm{L}, \dagger, \mathcal{X}}^{\star, \text { sem }}$, where $\dagger \in\{$ set, dir, con $\}$, sem $\in \mathrm{CMP}$ and $\star \in\{\cap, \cap, \cup\}$. Thus, instead of explicitly mentioning the set $A$ of attack rules, we indicate (by $\dagger \in\{$ set, dir, con $\}$ ) what type of attack rules are taken into consideration.

In these notations, Theorem 2 indicates that for every fixed $\dagger \in\{$ dir, con, set $\}$ and $\star \in\{\cap, \cap, \cup\}$, we actually have two different argumentative entailments of the form $\sim_{L, \dagger, \mathcal{X}}^{\star, \text { sem }}:$ one for sem $\in \mathrm{ME}$ and the other one for sem $\in \mathrm{SE}$. This is formalized in the next corollary.

Corollary 1. Let $\dagger \in\{$ dir, con, set $\}$ and $\star \in\{\cap, \cap, \cup\}$. For every sem $\in$ ME the entailments $\sim_{\mathrm{L}, \dagger, \mathcal{X}}^{\star}$ are the same. Likewise, for all sem $\in \mathrm{SE}, \sim_{\mathrm{L}, \dagger, \mathcal{X}}^{\star, \mathrm{sem}}$ coincide.

Note 4. To simplify the presentation, i.e., in order to avoid too many case distinctions, we have not listed $\mathrm{cmp}$ among the semantics in SE and in ME. However, we note that $\sim_{\mathrm{L}, \dagger, \mathcal{X}}^{\star, \mathrm{cmp}}=\sim_{\mathrm{L}, \dagger, \mathcal{X}}^{\star, \text { sem }}$ either when $\star=\cup$ and sem $\in \mathrm{ME}$, or when $\star \in\{\cap$, ก $\}$ and sem $\in$ SE. Thus, implicitly, reasoning with sem $=\mathrm{cmp}$ is covered (here and in what follows) as well.

The next results relate argumentative entailments and consistency-based ones. The first result shows a correspondence between argumentative entailments and inference by maximally consistent sets of the premises (Rescher and Manor 1970; Makinson 2003) (see also (Arieli, Borg, and Straßer 2018) and (Arieli, Borg, and Heyninck 2019)). For this, we recall the following entailments:

Definition 9 (MCS-based entailments). Let $L=\langle\mathcal{L}, \vdash\rangle$ be a logic, $\mathcal{S}$ a set of $\mathcal{L}$-formulas and $\mathcal{X}$ a $\vdash$-consistent set of $\mathcal{L}$-formulas. The following entailments are defined in a way similar to those in Definition 6:

- $\mathcal{S} \sim_{\mathrm{L}, \mathcal{X}}^{\cap, \mathrm{mcs}} \phi$ iff $\phi \in \mathrm{CN}_{\mathrm{L}}\left(\bigcap \operatorname{MCS}_{\mathrm{L}}^{\mathcal{X}}(\mathcal{S}) \cup \mathcal{X}\right)$

- $\mathcal{S} \sim_{\mathrm{L}, \mathcal{X}}^{\mathrm{n}, \mathrm{mcs}} \phi$ iff $\phi \in \bigcap_{\mathcal{T} \in \operatorname{MCS}_{\mathrm{L}}^{\mathcal{X}}(\mathcal{S})} \operatorname{CN}_{\mathrm{L}}(\mathcal{T} \cup \mathcal{X})$

- $\mathcal{S} \sim_{\mathrm{L}, \mathcal{X}}^{\cup, \mathrm{mcs}} \phi$ iff $\phi \in \bigcup_{\mathcal{T} \in \operatorname{MCS}_{\mathrm{L}}^{\mathcal{X}}(\mathcal{S})} \mathrm{CN}_{\mathrm{L}}(\mathcal{T} \cup \mathcal{X})$
Note 5. The family of entailments in Definition 9 covers, for instance, those proposed under the name default assumptions in (Makinson 2003) or (in view of the characterization in (Van De Putte 2013)) adaptive logics (Batens 2007; Straßer 2014) that have lower limit logics with adequate sequent calculi of the type in Figure 2.

We now relate the entailments in Definitions 6 and 9. A result similar to the next proposition is given in (Arieli, Borg, and Heyninck 2019), but only for DUcut $\emptyset$ and ConUcut $\emptyset$ as the attack rules and just for sem $\in$ $\{$ grd, prf, stb $\}$. Here we generalize the setting to every logic in which the rules from Figure 2 are admissible, every completeness-based semantics, and every framework whose attacks are of type dir, con or set.

Theorem 3. The following equivalences hold:

1. $\mathcal{S} \sim_{\mathrm{L}, \dagger, \mathcal{X}}^{\cup \text {,sem }} \psi$ iff $\mathcal{S} \sim_{\mathrm{L}, \mathcal{X}}^{\cup \text { mcs }} \psi$ for every $\dagger \in\{$ con, set, dir $\}$ and sem $\in \mathrm{ME} \cup\{\mathrm{cmp}\}$.

2. $\mathcal{S} \sim_{\mathrm{L}, \dagger, \mathcal{X}}^{\cap, \text { sem }} \psi$ iff $\mathcal{S} \sim_{\mathrm{L}, \mathcal{X}}^{\cap, \text { mcs }} \psi$ for every $\dagger \in\{$ con, set $\}$ and sem $\in$ CMP.

3. $\mathcal{S} \sim_{\mathrm{L}, \dagger, \mathcal{X}}^{\cap, \text { sem }} \psi$ iff $\mathcal{S} \sim_{\mathrm{L}, \mathcal{X}}^{\cap, \text { mcs }} \psi$ for $\dagger=\operatorname{dir}$ and sem $\in \mathrm{ME}$.

4. $\mathcal{S} \sim_{\mathrm{L}, \dagger, \mathcal{X}}^{\mathrm{n}, \mathrm{sem}} \psi$ iff $\mathcal{S} \sim_{\mathrm{L}, \mathcal{X}}^{\mathrm{n}, \mathrm{mcs}} \psi$ for every $\dagger \in\{$ con, dir $\}$ and sem $\in$ ME.

5. $\mathcal{S} \sim_{\mathrm{L}, \dagger, \mathcal{X}}^{\mathrm{n}, \mathrm{X}} \psi$ iff $\mathcal{S} \sim_{\mathrm{L}, \mathcal{X}}^{\cap, \mathrm{mcs}} \psi$ for every $\dagger \in\{$ con, set $\}$ and sem $\in \mathrm{SE} \cup\{\mathrm{cmp}\}$.

Proof outline. Item 1: Suppose first that $\mathcal{S} \sim_{\mathrm{L}, \mathcal{X}}^{\cup, \mathrm{mcs}} \psi$. Thus, there is a maximal $\vdash \mathcal{X}$-consistent subset $\mathcal{T}$ of $\mathcal{S}$ for which $\mathcal{T}, \mathcal{X} \vdash \psi$. It can be shown (see, e.g., (Arieli, Borg, and Straßer 2020, Lemma 10)) that $\operatorname{Arg}_{\mathrm{L}}^{\mathcal{X}}(\mathcal{T}) \in \operatorname{Ext}_{\mathrm{cmp}}(\mathcal{A F})$. Since $\mathrm{L}$ is finitary and by the completeness of $\mathrm{C}$, there is an argument $\Gamma \Rightarrow \psi \in \operatorname{Arg}_{\mathrm{L}}^{\mathcal{X}}(\mathcal{T})$. Thus, $\mathcal{S} \sim_{\mathrm{L}, \dagger, \mathcal{X}}^{\cup \text { sem }} \psi$.

For the converse, suppose that $\mathcal{S} \sim_{\mathrm{L}, \dagger, \mathcal{X}}^{\cup, \mathrm{ise}} \psi$. Thus, there is an $\mathcal{E} \in \operatorname{Ext}_{\mathrm{cmp}}(\mathcal{A F})$ with a $\Gamma \Rightarrow \psi \in \mathcal{E}$. It can be shown (see, e.g., (Arieli, Borg, and Straßer 2020, Lemma 4)) that $\Gamma \backslash \mathcal{X}$ is a $\vdash \mathcal{X}$-consistent subset of $\mathcal{S}$. By the soundness of $\mathrm{C}, \Gamma \vdash \psi$. Thus, there is a maximal $\vdash \mathcal{X}$-consistent subset $\mathcal{T}$ of $\mathcal{S}$ for which $\mathcal{T}, \mathcal{X} \vdash \psi$, and so $\mathcal{S} \sim_{\mathrm{L}, \mathcal{X}}^{\cup} \psi$.

Item 2 for ME-semantics and Item 3 follow from the fact that $\operatorname{Free}^{\mathcal{X}}(\mathcal{S})=\bigcap \operatorname{MCS}^{\mathcal{X}}(\mathcal{S})$, together with the fact that if $\mathcal{A F}$ is of type dir or con, and if sem $\in \mathrm{ME}$, then $\operatorname{Ext}_{\text {sem }}(\mathcal{A F})=\left\{\operatorname{Arg}_{\mathrm{L}}^{\mathcal{X}}(\mathcal{T}) \mid \mathcal{T} \in \operatorname{MCS}_{\mathrm{L}}^{\mathcal{X}}(\mathcal{S})\right\}$. Item 2 for sem $\in$ SE follows from Item 3 of Theorem 1 . Item 4 is a straightforward generalization to every ME-semantics of a similar result shown in (Arieli, Borg, and Straßer 2020) for sem $\in\{$ prf, stb $\}$. Item 5 follows from Item 2 , as for singleextension semantics, $\sim_{\mathrm{L}, \dagger, \mathcal{X}}^{\mathrm{n}, \text { sem }}$ and $\sim_{\mathrm{L}, \dagger, \mathcal{X}}^{\cap, \text { sem }}$ coincide (concerning sem $=\mathrm{cmp}$, this follows from Note 4 ).

Example 4. Consider the sets $\mathcal{S}=\{q, p \supset \square r, q \supset \square \neg r\}$ and $\mathcal{X}=\{p\}$ from Example 1. We have that: $\operatorname{MCS}_{\mathrm{S} 4}^{\{p\}}(\mathcal{S})=$

$$
\{\{q, p \supset \square r\},\{q, q \supset \square \neg r\},\{p \supset \square r, q \supset \square \neg r\}\} .
$$

Thus, $\mathcal{S} \mathcal{L}_{\mathrm{s} 4, \mathrm{dir},\{p\}}^{\star, \text { mcs }} \psi$ for any $\psi \in \mathcal{S}$ and $\star \in\{\cap$, ก $\}$, while $\mathcal{S} \sim_{\mathrm{S} 4, \operatorname{dir},\{p\}}^{\star, \operatorname{mcs}}(p \supset \square r) \vee(q \supset \square \neg r)$ where $\star \in\{\cap, \cup\}$. By 
Theorem 3, it is not a coincidence that these are the same results as those in Example 2, obtained for any sem $\in \mathrm{ME}$ of the framework in Example 1 (and Figure 1).

To complete the characterization in Theorem 3 to the other entailments, we need the following notations:

Definition 10 ( $\Omega$-entailments). Let $\mathrm{L}=\langle\mathcal{L}, \vdash\rangle$ be a logic. For sets $\mathcal{S}, \mathcal{X}$ of $\mathcal{L}$-formula, $\Omega=\Omega^{\mathcal{X}}(\mathcal{S})$ denotes the set that is considered in the second bullet of Notation 2. We define:

- $\mathcal{S} \sim_{\mathrm{L}, \mathcal{X}}^{\cap, \Omega} \phi$ iff $\phi \in \mathrm{CN}_{\mathrm{L}}\left(\bigcap_{\omega \in \Omega} \bigcap_{\mathcal{T} \in \omega} \mathcal{T} \cup \mathcal{X}\right)$.

- $\mathcal{S} \sim_{\mathrm{L}, \mathcal{X}}^{\mathrm{\Pi}, \Omega} \phi$ iff $\phi \in \bigcap_{\omega \in \Omega} \bigcup_{\mathcal{T} \in \omega} \mathrm{CN}_{\mathrm{L}}(\mathcal{T} \cup \mathcal{X})$.

- $\mathcal{S} \sim_{\mathrm{L}, \mathcal{X}}^{\cup, \Omega} \phi$ iff $\phi \in \bigcup_{\omega \in \Omega} \bigcup_{\mathcal{T} \in \omega} \mathrm{CN}_{\mathrm{L}}(\mathcal{T} \cup \mathcal{X})$.

The following result immediately follows from Definition 10 and Item 2 of Theorem 1.

Theorem 4. For $\dagger=$ set, sem $\in M E$, and $\star \in\{\cap, \cap, \cup\}$, it holds that $\mathcal{S} \sim_{\mathrm{L}, \dagger, \mathcal{X}}^{\star, \mathrm{sem}} \psi$ iff $\mathcal{S} \sim_{\mathrm{L}, \mathcal{X}}^{\star, \Omega} \psi$.

\section{Entailments Classifications}

We now examine properties of the entailment relations. We distinguish between properties that are concerned with nonmonotonic reasoning and those for inconsistency handling.

\subsection{Principles for Nonmonotonic Reasoning}

Monotonicity is a substantial characteristic of a reasoning process, as it determines whether inferences remain valid in the presence of new information. In this section we check which argumentative entailments have this property, and those who lack it are examined with respect to common patterns of nonmonotonic reasoning that have been identified and studied in the literature (see, e.g., (Gabbay 1985; Shoham 1988; Kraus, Lehmann, and Magidor 1990; Lehmann and Magidor 1992; Makinson 1994)).

First, we show that credulous entailments with respect to multiple extension semantics are in fact monotonic.

Proposition 2. Every entailment of the form $\sim_{\mathrm{L}, \dagger, \mathcal{X}}^{\cup \text {,sem }}$, where sem $\in \mathrm{ME}$ and $\dagger \in\{\mathrm{dir}$, con, set $\}$, is monotonic.

Proof. We show that $\sim_{L, \mathcal{X}}^{\cup, \text { mcs }}$ is monotonic, and so the proposition follows by Item 1 of Theorem 3 .

Suppose that $\mathcal{S} \sim_{\mathrm{L}, \mathcal{X}}^{\cup \text {,mcs }} \psi$. Then there is a maximal $\vdash_{\mathcal{X}}$ consistent subset $\mathcal{T}$ of $\mathcal{S}$ for which $\mathcal{T}, \mathcal{X} \vdash \psi$. Consider $\mathcal{S} \cup \mathcal{S}^{\prime}$. Clearly, $\mathcal{T}$ is a $\vdash \mathcal{X}$-consistent subset of $\mathcal{S} \cup \mathcal{S}^{\prime}$, and so there is a maximal $\vdash \mathcal{X}$-consistent subset $\mathcal{T}^{\prime}$ of $\mathcal{S} \cup \mathcal{S}^{\prime}$ such that $\mathcal{T} \subseteq \mathcal{T}^{\prime}$. By the monotonicity of $\vdash$, we have: $\mathcal{T}^{\prime}, \mathcal{X} \vdash \psi$, thus $\mathcal{S}, \mathcal{S}^{\prime} \sim_{\mathrm{L}, \mathcal{X}}^{\cup \text { ms }} \psi$.

Note 6. The last proposition does not hold for sem $\in \mathrm{SE}$. Indeed, $\operatorname{Ext}_{\text {sem }}\left(\mathcal{A F}_{\mathrm{CL}, \mathrm{A}}^{\emptyset}(\{p\})\right)=\left\{\operatorname{Arg}_{\mathrm{L}}(p)\right\}$ for every $\mathrm{A}$ and sem, while $\operatorname{Ext}_{\text {sem }}\left(\mathcal{A F}_{\mathrm{CL}, \mathrm{A}}^{\emptyset}(\{p, \neg p\})\right)=\left\{\operatorname{Arg}_{\mathrm{L}}(\emptyset)\right\}$ for any sem $\in \mathrm{SE}$. Thus, for such sem we have that for every $\dagger \in\{\operatorname{dir}$, con, set $\}, p \underset{\mathrm{L}, \dagger, \emptyset}{\cup, \text { sem }} p$ while, $p, \neg p \mathcal{L}_{\mathrm{L}, \dagger, \emptyset}^{\cup, \text { sem }} p$.

We now turn to nonmonotonic entailments. It is common to examine such entailments according to the following properties:
Definition 11. Let $L=\langle\mathcal{L}, \vdash\rangle$ be a propositional logic and let $\sim \subseteq \wp(\operatorname{WFF}(\mathcal{L})) \times \operatorname{WFF}(\mathcal{L})$. We say that $\sim$ satisfies:

$\vdash$-cautious reflexivity $(\vdash-\mathrm{cR}): \phi \sim \phi$ for a $\vdash$-consistent $\phi$.

$\vdash$-right weakening ( $\vdash-\mathrm{RW}): \mathcal{S} \sim \phi$ and $\phi \vdash \psi$ imply $\mathcal{S} \sim \psi$.

$\vdash$-left logical equivalence ( $\vdash$-LLE): If $\mathcal{S}, \phi \sim \sigma, \psi \vdash \phi$, and $\phi \vdash \psi$, then $\mathcal{S}, \psi \sim \sigma$.

cautious monotonicity (CM): If $\mathcal{S} \sim \phi$ and $\mathcal{S} \sim \psi$, then $\mathcal{S}, \phi \sim \psi$.

cautious cut (CC): If $\mathcal{S} \sim \psi$ and $\mathcal{S}, \psi \sim \phi$, then $\mathcal{S} \sim \phi$. or (Or): If $\mathcal{S}, \phi \sim \sigma$ and $\mathcal{S}, \psi \sim \sigma$, then $\mathcal{S}, \phi \vee \psi \sim \sigma$.

rational monotonicity (RM): If $\mathcal{S} \sim \psi$ and $\mathcal{S} \not \psi \neg \phi$, then $\mathcal{S}, \phi \sim \psi$.

Since the rule Or is defined in terms of a disjunction connective $V$, in what follows we suppose that, in addition to the rules in Figure 2, the two rules in Figure 3 are part of (or admissible in) the sequent calculus $C$.

$[\vee \Rightarrow] \quad \frac{\Gamma, \phi \Rightarrow \Delta \quad \Gamma, \psi \Rightarrow \Delta}{\Gamma, \phi \vee \psi \Rightarrow \Delta} \quad[\Rightarrow \vee] \quad \frac{\Gamma \Rightarrow \phi, \psi, \Delta}{\Gamma \Rightarrow \phi \vee \psi, \Delta}$

Figure 3: Rules for disjunction

The properties in Definition 11 are often gathered for classifying systems for nonmonotonic inference.

Definition 12. We say that an entailment $\sim$ is:

- $\vdash$-cumulative, if it satisfies $\vdash$-cR, $\vdash$-RW, $\vdash$-LLE, CM, CC.

- $\vdash$-preferential, if it is $\vdash$-cumulative and satisfies Or.

- $\vdash$-rational, if it is $\vdash$-preferential and satisfies RM.

Table 1 summarizes the classification of the argumentative entailments according to their components.

\begin{tabular}{l|c|c|c}
\hline & $\sim_{\mathrm{L}, \dagger, \mathcal{X}}^{\cap, \text { sem }}$ & $\sim_{\mathrm{L}, \dagger, \mathcal{X}}^{\mathrm{n}, \mathrm{sem}}$ & $\sim_{\mathrm{L}, \dagger, \mathcal{X}}^{\cup, \text { sem }}$ \\
\hline \hline cumulativity, $\dagger \in\{$ con, dir $\}$ & $\mathrm{CMP}$ & $\mathrm{CMP}$ & $\mathrm{SE}$ \\
\hline cumulativity, $\dagger=$ set & $\mathrm{CMP}$ & $\mathrm{SE}^{*}$ & $\mathrm{SE}$ \\
\hline \hline preferentiality, $\dagger=\mathrm{con}$ & - & $\mathrm{ME}$ & - \\
\hline preferentiality, $\dagger=\mathrm{dir}$ & $\mathrm{SE}^{*}$ & $\mathrm{CMP}$ & $\mathrm{SE}$ \\
\hline preferentiality, $\dagger=$ set & - & - & - \\
\hline \hline rationality, $\dagger \in\{$ set, con $\}$ & - & - & - \\
\hline rationality, $\dagger=$ dir & $\mathrm{SE}^{*}$ & $\mathrm{SE}^{*}$ & $\mathrm{SE}$ \\
\hline \hline monoton., $\dagger \in\{$ dir, con, set $\}$ & - & - & $\mathrm{ME}^{*}$ \\
\hline
\end{tabular}

Table 1: Summary of the properties of nonmonotonic inference. Cells with an asterisk apply also to sem $=\mathrm{cmp}$ (see Note 4 ).

We leave the proofs of the positive parts of Table 1 to the full paper. Below, we show some negative results in terms of counter-examples, all of them are considered w.r.t. $\mathrm{L}=\mathrm{CL}$.

Concerning cumulativity, the next example shows that CC is violated for $\sim_{\mathrm{L}, \mathrm{set}, \emptyset}^{\mathrm{n}, \mathrm{sem}}$ when sem $\in \mathrm{ME}$. 
Example 5. Let $\mathcal{S}=\left\{\psi_{1}, \psi_{2}, \psi_{3}\right\}$, where $\psi_{1}=p \wedge s$, $\psi_{2}=q \wedge(s \supset t)$, and $\psi_{3}=\neg(p \wedge q) \wedge(q \supset s) \wedge(s \supset t)$. For every $\mathcal{S}$-based framework with set-attacks, we have the following stable (preferred, semi-stable, stage) extensions: $\mathcal{E}_{1}=\operatorname{Arg}_{\mathrm{CL}}\left(\left\{\psi_{1}, \psi_{2}\right\}\right)$,

$\mathcal{E}_{2}=\operatorname{Arg}_{\mathrm{CL}}\left(\left\{\psi_{2}, \psi_{3}\right\}\right)$,

$\mathcal{E}_{3}=\operatorname{Arg}_{\mathrm{CL}}\left(\left\{\psi_{1}, \psi_{3}\right\}\right)$,

$\mathcal{E}_{4}=\operatorname{Arg}_{\mathrm{CL}}\left(\left\{\psi_{1}\right\}\right) \cup \operatorname{Arg}_{\mathrm{CL}}\left(\left\{\psi_{2}\right\}\right) \cup \operatorname{Arg}_{\mathrm{CL}}\left(\left\{\psi_{3}\right\}\right)$.

Therefore, $\mathcal{S} \sim_{\mathrm{CL}, \text { set }, \emptyset}^{\text {ח, sem }} s$ but $\mathcal{S} \mid \mathcal{L}_{\mathrm{CL}, \text { set }, \emptyset}^{\mathrm{In}, \text { sem }} t$.

We now let $\mathcal{S}^{\prime}=\mathcal{S} \cup\{s\}$. This time, the stable (preferred, semi-stable, stage) extensions are:

$\mathcal{E}_{1}^{\prime}=\operatorname{Arg}_{\mathrm{CL}}\left(\left\{\psi_{1}, \psi_{2}, s\right\}\right)$,

$\mathcal{E}_{2}^{\prime}=\operatorname{Arg}_{\mathrm{CL}}\left(\left\{\psi_{2}, \psi_{3}, s\right\}\right)$,

$\mathcal{E}_{3}^{\prime}=\operatorname{Arg}_{\mathrm{CL}}\left(\left\{\psi_{1}, \psi_{3}, s\right\}\right)$,

$\mathcal{E}_{4}^{\prime}=\operatorname{Arg}_{\mathrm{CL}}\left(\left\{\psi_{1}, s\right\}\right) \cup \operatorname{Arg}_{\mathrm{CL}}\left(\left\{\psi_{2}, s\right\}\right) \cup \operatorname{Arg}_{\mathrm{CL}}\left(\left\{\psi_{3}, s\right\}\right)$.

Therefore, $\mathcal{S}^{\prime} \sim_{\text {CL,set }, \emptyset}^{\text {In,sem }} t$.

The next example, taken from (Benferhat, Dubois, and Prade 1997), shows that CC is also violated by $\sim_{L, \emptyset}^{\cup, m c s}$. Thus, by Theorem 3 it follows that cumulativity, preferentiality and rationality are violated by $\sim_{L, \dagger, \emptyset}^{\cup, \text { sem }}$ for every $\dagger \in\{$ con, set, dir $\}$ and sem $\in$ ME.

Example 6. Let $\mathcal{S}=\{p \wedge q, \neg p \wedge r\}$. Then $\operatorname{MCS}_{\mathrm{CL}}^{\emptyset}(\mathcal{S})=$ $\{\{p \wedge q\},\{\neg p \wedge r\}\}$, and so $\mathcal{S} \sim_{\mathrm{CL}, \emptyset}^{\cup, \text { mcs }} q$, but $\mathcal{S} \mid \mathcal{L}_{\mathrm{CL}, \emptyset}^{\cup, \text { mcs }} q \wedge r$. Let now $\mathcal{S}^{\prime}=\mathcal{S} \cup\{q\}$. In this case $\operatorname{MCS}_{\mathrm{CL}}^{\emptyset}\left(\mathcal{S}^{\prime}\right)=\{\{p \wedge$ $q, q\},\{\neg p \wedge r, q\}\}$, thus $\mathcal{S}^{\prime} \sim_{\mathrm{CL}, \emptyset}^{\cup \text {,mcs }} q \wedge r$.

Next we show that Or fails for $\sim_{L, \emptyset}^{\cap, m c s}$. It follows, then, (by Items 2 and 3 of Theorem 3 ) that $\sim_{L, \dagger, \emptyset}^{\cap, \text { sem }}$ is not preferential when $\dagger \in\{$ con, set $\}$ or when $\dagger=$ dir and sem $\in$ ME. By the same example (together with Item 5 of Theorem 3), $\sim_{L, \dagger, \emptyset}^{\text {n,sem }}$ is not preferential also when $\dagger \in\{$ con, set $\}$ and sem $\in$ SE.

Example 7. Let $\mathcal{S}=\{\neg p, \neg q, \neg p \supset r, \neg q \supset r\}$. Then:

- $\mathcal{S}, p \sim_{\mathrm{CL}, \emptyset}^{\cap, \mathrm{mcs}} r$, since $\operatorname{MCS}_{\mathrm{CL}}^{\emptyset}(\mathcal{S} \cup\{p\})=\{\{p, \neg q, \neg p \supset$ $r, \neg q \supset r\},\{\neg p, \neg q, \neg p \quad \supset \quad r, \neg q \quad \supset r\}\}$ and so $\bigcap \operatorname{MCS}_{\mathrm{CL}}^{\emptyset}(\mathcal{S} \cup\{p\})=\{\neg q, \neg p \supset r, \neg q \supset r\}$,

- $\mathcal{S}, q \sim_{\mathrm{CL}, \emptyset}^{\cap, \text { mcs }} r$, since $\operatorname{MCS}_{\mathrm{CL}}^{\emptyset}(\mathcal{S} \cup\{q\})=\{\{\neg p, q, \neg p \supset$ $r, \neg q \supset r\},\{\neg p, \neg q, \neg p \quad \supset \quad r, \neg q \quad \supset r\}\}$ and so $\bigcap \operatorname{MCS}_{\mathrm{CL}}^{\emptyset}(\mathcal{S} \cup\{q\})=\{\neg p, \neg p \supset r, \neg q \supset r\}$,

- $\mathcal{S}, p \vee q \quad \mathcal{W}_{\mathrm{CL}, \emptyset}^{\cap, \mathrm{mcs}} r$, since $\operatorname{MCS}_{\mathrm{CL}}^{\emptyset}(\mathcal{S} \cup\{p \vee q\})=$ $\{\{p \vee q, \neg p, \neg p \supset r, \neg q \supset r\},\{p \vee q, \neg q, \neg p \supset r, \neg q \supset$ $r\},\{\neg p, \neg q, \neg p \supset r, \neg q \supset r\}\}$, and so $\bigcap \operatorname{MCS}_{\mathrm{CL}}^{\emptyset}(\mathcal{S} \cup$ $\{p \vee q\})=\{\neg p \supset r, \neg q \supset r\}$.

As Table 1 shows, rationality fails for $\sim_{L, \dagger, \mathcal{X}}^{\star, \text { sem }}$ for $\star \epsilon$ $\{\cap, \cap\}$, unless $\dagger=$ dir and sem $=$ SE. E.g., by the next example and Theorem 3, RM is violated by entailments of the form $\sim_{L, \dagger, \emptyset}^{\text {n,sem }}$ where $\dagger \in\{$ con, dir $\}$ and sem $\in$ ME.

Example 8. We show that RM doesn't hold for $\sim_{L, \emptyset}^{\mathrm{n}, \mathrm{mcs}}$. For this, consider again classical logic as the base logic and let $\mathcal{S}=\{r, p \wedge q \wedge \neg r,(p \wedge r) \supset \neg q, \neg p \wedge q\}$. We have: $\operatorname{MCS}_{\mathrm{CL}}^{\emptyset}(\mathcal{S})=\{\{r,(p \wedge r) \supset \neg q, \neg p \wedge q\},\{p \wedge q \wedge \neg r,(p \wedge$ $r) \supset \neg q\}$. Only one of the two sets in $\operatorname{MCS}_{\mathrm{CL}}^{\emptyset}(\mathcal{S})$ implies $\neg p$, while both of them imply $q$. Thus, $\mathcal{S} \sim_{\mathrm{L}, \emptyset}^{\mathrm{n}, \mathrm{mcs}} q$ and $\mathcal{S} \mathscr{W}_{\mathrm{L}, \emptyset}^{\mathrm{m}, \mathrm{mcs}} \neg p$. Now, $\operatorname{MCS}_{\mathrm{CL}}^{\emptyset}(\mathcal{S} \cup\{p\})=\{\{r,(p \wedge r) \supset$ $\neg q, \neg p \wedge q\},\{p \wedge q \wedge \neg r,(p \wedge r) \supset \neg q, p\},\{r, p,(p \wedge r) \supset$ $\neg q\}$ \}. It follows that $\mathcal{S}, p \mathcal{H}_{\mathrm{L}, \emptyset}^{\mathrm{R}, \mathrm{mcs}} q$, and so RM is violated.

\subsection{Principles for Inconsistency Handling}

A primary goal of argumentation theory is to tolerate conflicting arguments. Such arguments may originate from contradictory information, thus argumentative entailments (unlike classical logic entailment, for instance) should not be 'explosive', namely: an inconsistent set of premises should not imply any conclusion whatsoever. In this section we study different properties for rational reasoning in the presence of inconsistency. We start by introducing some notations and notions:

- We denote by $\mathcal{S}_{1} \| \mathcal{S}_{2}$ that the sets $\mathcal{S}_{1}$ and $\mathcal{S}_{2}$ are syntactically disjoint, that is, $\operatorname{Atoms}\left(\mathcal{S}_{1}\right) \cap \operatorname{Atoms}\left(\mathcal{S}_{2}\right)=\emptyset$.

- We say that a set $\mathcal{S}$ s.t. $\operatorname{Atoms}(\mathcal{S}) \subsetneq \operatorname{Atoms}(\mathcal{L})$ is contaminating (w.r.t. $\sim \mathcal{X}$ ), if for every $\mathcal{S}^{\prime}$ s.t. $\mathcal{S} \cup \mathcal{X} \| \mathcal{S}^{\prime}$ and every $\mathcal{L}$-formula $\phi$, it holds that $\mathcal{S} \sim \mathcal{X} \phi$ iff $\mathcal{S}, \mathcal{S}^{\prime} \sim_{\mathcal{X}} \phi$.

Definition 13. Let $\mathcal{X} \subseteq \operatorname{WFF}(\mathcal{L})$ be $\vdash$-consistent, $\sim_{\mathcal{X}} \subseteq$ $\wp(\operatorname{WFF}(\mathcal{L})) \times \operatorname{WFF}(\mathcal{L})$, and $\vdash \subseteq \wp(\operatorname{WFF}(\mathcal{L})) \times \operatorname{WFF}(\overline{\mathcal{L}})$ be a consequence relation in the sense of Definition 1 . The following are properties that $\sim_{\mathcal{X}}$ may satisfy:

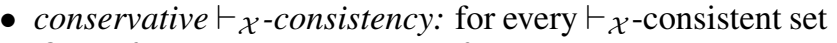
$\mathcal{S}$ of $\mathcal{L}$-formulas and every $\mathcal{L}$-formula $\psi$ it holds that $\mathcal{S} \sim \mathcal{X} \psi$ iff $\mathcal{S} \cup \mathcal{X} \vdash \psi$.

- paraconsistency (da Costa 1974): for every distinct $p, q \in$ Atoms $(\mathcal{L})$ it holds that $p, \neg p \not \mathcal{W}_{\emptyset} q$.

- non-interference (Caminada, Carnielli, and Dunne 2011): for every two sets $\mathcal{S}_{1}, \mathcal{S}_{2}$ of $\mathcal{L}$-formulas, and every $\mathcal{L}$ formula $\phi$ such that $\mathcal{S}_{1} \cup\{\phi\} \cup \mathcal{X} \| \mathcal{S}_{2}$, it holds that $\mathcal{S}_{1} \sim \mathcal{X} \phi$ iff $\mathcal{S}_{1}, \mathcal{S}_{2} \sim \mathcal{X} \phi$.

- crash-resistance (Caminada, Carnielli, and Dunne 2011): there is no $\sim \mathcal{X}$-contaminating set of $\mathcal{L}$-formulas.

For non-interference we need the following definition:

Definition 14 (uniformity). A logic $L=\langle\mathcal{L}, \vdash\rangle$ is said to be uniform (Łos and Suzsko 1958; Urquhart 2001), if for every two sets of $\mathcal{L}$-formulas $\mathcal{S}_{1}, \mathcal{S}_{2}$ and a formula $\phi$ such that $\mathcal{S}_{2}$ is both $\vdash$-consistent and syntactically disjoint from $\mathcal{S}_{1} \cup\{\phi\}$, it holds that $\mathcal{S}_{1} \vdash \phi$ iff $\mathcal{S}_{1}, \mathcal{S}_{2} \vdash \phi$.

Table 2 summarizes the results concerning inconsistency handling properties of argumentative entailments.

Below are proofs for some of the results in Table 2. We start with conservative consistency.

Proposition 3. Let $L=\langle\mathcal{L}, \vdash\rangle$ be a logic. For every $\star \in\{\cap, \cap, \cup\}$, sem $\in C M P$ and $\dagger \in\{$ set, dir, con $\}$, the entailment $\sim_{\mathrm{L}, \dagger, \mathcal{X}}^{\star, \text { sem }}$ satisfies conservative $\vdash_{\mathcal{X}}$-consistency.

Proof. Let $\mathcal{S}$ be a $\vdash \mathcal{X}$-consistent set of $\mathcal{L}$-formulas. Suppose first that there is an $a \in \operatorname{Arg}_{\mathrm{L}}^{\mathcal{X}}(\mathcal{S})$ such that some $b \in \operatorname{Arg}_{\mathrm{L}}^{\mathcal{X}}(\mathcal{S})$ attacks $a$. Thus Conc $(b) \Rightarrow \neg \phi$ is derivable, where $\phi \in \operatorname{Supp}(a)$ for $\dagger=\operatorname{dir}$ and $\dagger=$ con, and 


\begin{tabular}{l|c|c|c}
\hline & $\sim_{\mathrm{L}, \dagger, \mathcal{X}}^{\cap, \text { sem }}$ & $\sim_{\mathrm{L}, \dagger, \mathcal{X}}^{\mathrm{n}, \text { sem }}$ & $\sim_{\mathrm{L}, \dagger, \mathcal{X}}^{\cup, \text { sem }}$ \\
\hline \hline conservative $\vdash_{\mathcal{X}}$-consistency & $\mathrm{CMP}$ & $\mathrm{CMP}$ & $\mathrm{CMP}$ \\
\hline \hline paraconsistency, $\mathcal{X}=\emptyset$ & $\mathrm{CMP}$ & $\mathrm{CMP}$ & $\mathrm{CMP}$ \\
\hline \hline non-interference, $\dagger \in\{$ con, set $\}$ & $\mathrm{CMP}$ & $\mathrm{CMP}$ & $\mathrm{ME}^{*}$ \\
\hline non-interference, $\dagger=\mathrm{dir}$ & $\mathrm{ME}$ & $\mathrm{ME}$ & $\mathrm{ME}^{*}$ \\
\hline \hline crash-resistance, $\dagger \in\{$ con, set $\}$ & $\mathrm{CMP}$ & $\mathrm{CMP}$ & $\mathrm{ME}^{*}$ \\
\hline crash-resistance, $\dagger=$ dir & $\mathrm{ME}$ & $\mathrm{ME}$ & $\mathrm{ME}^{*}$ \\
\hline \hline
\end{tabular}

Table 2: Summary of the results about inconsistency handling. For non-interference and crash-resistance $L$ is assumed to be uniform. By Note 4, cells with an asterisk apply also to sem $=\mathrm{cmp}$.

$\phi=\bigwedge \operatorname{Supp}(a)$ for $\dagger=$ set (note that $b$ is not a ConUcutattacker, since $\mathcal{S}$ is $\vdash_{\mathcal{X}}$-consistent). Thus, by [Cut], we derive $\operatorname{Supp}(b) \Rightarrow \neg \phi$. By $[\neg \Rightarrow]$, the fact that $\phi \Rightarrow \neg \neg \phi$ is Cderivable, and [Cut], we derive $\operatorname{Supp}(b), \phi \Rightarrow$. So, by $[\wedge \Rightarrow]$ and $[\Rightarrow \neg]$, the sequent $\Rightarrow \neg(\wedge \operatorname{Supp}(b) \wedge \phi)$ is derivable, a

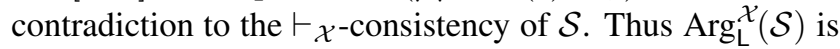
conflict-free, and so, $\operatorname{Ext}_{\text {sem }}\left(\mathcal{A} \mathcal{F}_{\mathrm{L}, \mathrm{A}}^{\mathcal{X}}(\mathcal{S})\right)=\left\{\operatorname{Arg}_{\mathrm{L}}^{\mathcal{X}}(\mathcal{S})\right\}$ for every sem $\in$ CMP.

Let now $\Gamma \Rightarrow \phi \in \operatorname{Arg}_{\mathrm{L}}^{\mathcal{X}}(\mathcal{S})$. Then $\Gamma \vdash \phi$ by the soundness of $\mathrm{C}$, and by the monotonicity of $\vdash$ (Def. 1$), \mathcal{S} \cup \mathcal{X} \vdash \phi$. Hence, $\sim_{\mathrm{L}, \dagger, \mathcal{X}}^{\star, \text { sem }} \subseteq \vdash_{\mathcal{X}}\left(\right.$ where $\left.\vdash_{\mathcal{X}}=\{(\mathcal{T}, \psi) \mid \mathcal{T} \cup \mathcal{X} \vdash \psi\}\right)$. Now, suppose that $\mathcal{S} \vdash \mathcal{X} \phi$. By the completeness of $\mathrm{C}$ for $\mathrm{L}$, and since $\mathrm{L}$ is finitary, there is $\Gamma \subseteq \mathcal{S}$, such that $\Gamma \Rightarrow$ $\phi \in \operatorname{Arg}_{\mathrm{L}}^{\mathcal{X}}(\mathcal{S})$. Since $\operatorname{Ext}_{\text {sem }}\left(\mathcal{A} \mathcal{F}_{\mathrm{L}, \mathrm{A}}^{\mathcal{X}}(\mathcal{S})\right)=\left\{\operatorname{Arg}_{\mathrm{L}}^{\mathcal{X}}(\mathcal{S})\right\}$ for every sem $\in$ CMP, we have that $\mathcal{S} \sim_{L, \dagger, \mathcal{X}}^{\star, \text { sem }} \phi$. Thus, $\vdash_{\mathcal{X}} \subseteq \sim_{\mathrm{L}, \dagger, \mathcal{X}}^{\star, \text { sem }}$. Altogether, $\vdash_{\mathcal{X}}=\sim_{\mathrm{L}, \dagger, \mathcal{X}}^{\star, \text { sem }}$.

As shown in Table 2, all the argumentative entailment relations considered here are paraconsistent. Below, we give a proof for cases where the attacks are of type set or con.

Proposition 4. For every sem $\in \mathrm{CMP}, \star \in\{\cap, \cap, \cup\}$ and $\dagger \in\{$ set, con $\}$, the entailment $\mathcal{L}_{\mathrm{L}, \dagger, \emptyset}^{\star, \mathrm{sem}}$ is paraconsistent.

Proof. Let sem be a completeness-based semantics, $\mathcal{S}=$ $\{p, \neg p\}$ and let $q \neq p$. There is no $a \in \operatorname{Arg}_{\mathrm{L}}(\{p\}) \cup$ $\operatorname{Arg}_{\mathrm{L}}(\{\neg p\})$ such that $\operatorname{Conc}(a)=q$ (otherwise, $a$ is either $\Rightarrow q$, or $p \Rightarrow q$, or $\neg p \Rightarrow q$, and by monotonicity and structurality we get that each case implies that $\neg p \vdash p$, which contradicts $\neg$ being a negation). Suppose that there is some $b \in \operatorname{Arg}_{\mathrm{L}}(\mathcal{S})$, such that $\operatorname{Conc}(b)=q$. Then $\operatorname{Supp}(b)=\mathcal{S}$. Note that one can derive the argument $\Rightarrow \neg(p \wedge \neg p)$ by [Ref], $[\neg \Rightarrow],[\wedge \Rightarrow]$ and $[\Rightarrow \neg]$ in $C$, and that this argument attacks $b$ by ConUcut, Ucut or Def. Thus, $b$ is attacked when $\dagger \in\{$ set, con $\}$ and it cannot be defended. Therefore, $\mathcal{S} \mid \mathcal{L}_{L, \uparrow, \emptyset}^{\star, \downarrow e m} q$.

Proofs of non-interference are rather lengthy, thus they are omitted. For the proofs, the base logic needs to be uniform (Definition 14), which is indeed the case for the vast majority of the logics considered in the literature. Under this assumption, in our setting (as defined in Section 4) noninterference implies crash-resistance.

As indicated in Table 2, the only skeptical entailments (i.e., when $\star \in\{\cap, \cap\}$ ) for which both non-interference and crash resistance are violated, are those where $\dagger=$ dir and sem $\in \mathrm{SE}$. We show this in the next example.

Example 9. Let $\mathcal{S}_{1}=\{p\}$ and $\mathcal{S}_{2}=\{q, \neg q\}$. It is easy to see that for every sem $\in \mathrm{SE}$ and $\star \in\{\cap, \cap\}$, it holds that $\mathcal{S}_{1} \sim_{\mathrm{L}, \mathrm{dir}, \emptyset}^{\star, \emptyset e m} p$ while $\mathcal{S}_{1}, \mathcal{S}_{2} \mid \mathcal{L}_{\mathrm{L}, \mathrm{dir}, \emptyset}^{\star, \mathrm{sem}} p$. Note also that $\{q, \neg q\}$ is a contaminating set in these cases.

\section{Discussion and Conclusion}

The primary goal of this paper is to provide an outlook on logic-based argumentative reasoning and its nature. We have done so in two senses:

a) Characterizations of Dung-type extensions according to the frameworks' components (Theorems 1 and 2) and accordingly identifications of the induced entailment relations (Theorems 3 and 4).

b) Classifications of the argumentative entailments according to the way they tolerate revised information and contradictory data (as summarized in Tables 1 and 2).

These results complete the picture, provided by some earlier works, to all the standard completeness-based Dung-style semantics and for the main types of attack rules. As a result, in addition to the obvious factors that affect the reasoning process (such as the base logic underlying the arguments at hand), we are now able to identify other factors, some of which were not so evident so far. Below we summarize some of our findings:

1. Theorem 1 vindicates the strong connection between argumentative inferences and reasoning with maximally consistent subsets. Yet, this correlation is kept either for multiple-extension semantics with attack rules of type dir or con, or for single-extension semantics with attack rules of type con or set. For the other combinations of semantics and attack rules the relation to reasoning with consistent subsets of the premises is more subtle.

2. By Corollary 1 , the primary consideration concerning the chosen semantics, in relation to the entailments that are induced by the underlying framework, is whether it allows a single extension or multiple extensions.

3. At least as far as plausible reasoning with inconsistent premises is concerned, Table 2 shows that multipleextension semantics are superior to single-extension ones. However, as shown in Table 1, only single-extension semantics with direct attack rules assure rational patterns of non-monotonic reasoning.

4. As expected, inferences are significantly affected by the type of reasoning under consideration (namely, credulous versus skeptical). For instance, only credulous reasoning with multiple-extension semantics yields monotonic entailments, while the other entailments are non-monotonic.

Future work involves, among others, the study of more expressive formalisms, based e.g. on first-order logics, and formalisms that incorporate priorities among arguments. 


\section{Acknowledgements}

This work is partially supported by the Israel Science Foundation (Grants No. 550/19).

\section{References}

Amgoud, L., and Besnard, P. 2010. A formal analysis of logic-based argumentation systems. In Proceedings of the 4th International Conference Scalable Uncertainty Management (SUM'10), volume 6379 of Lecture Notes in Computer Science, 42-55. Springer.

Amgoud, L., and Besnard, P. 2013. Logical limits of abstract argumentation frameworks. Journal of Applied NonClassical Logics 23(3):229-267.

Amgoud, L. 2014. Postulates for logic-based argumentation systems. International Journal of Approximate Reasoning 55(9):2028-2048.

Arieli, O., and Straßer, C. 2015. Sequent-based logical argumentation. Argument \& Computation 6(1):73-99.

Arieli, O., and Straßer, C. 2016. Deductive argumentation by enhanced sequent calculi and dynamic derivations. Electronic Notes in Theoretical Computer Science 323:21-37.

Arieli, O., and Straßer, C. 2019. Logical argumentation by dynamic proof systems. Theoretical Computer Science 781:63-91.

Arieli, O., and Straßer, C. 2020. On minimality and consistency tolerance in logical argumentation frameworks. In Proceedings of the 8th International Conference on Computational Models of Argument (COMMA'20), volume 326 of Frontiers in Artificial Intelligence and Applications, 91-102. IOS Press.

Arieli, O.; Borg, A.; and Heyninck, J. 2019. A review of the relations between logical argumentation and reasoning with maximal consistency. Annals of Mathematics and Artificial Intelligence 87(3):187-226.

Arieli, O.; Borg, A.; and Straßer, C. 2018. Reasoning with maximal consistency by argumentative approaches. Journal of Logic and Computation 28(7):1523-1563.

Arieli, O.; Borg, A.; and Straßer, C. 2020. Tuning logical argumentation frameworks: A postulate-derived approach. In Proceedings of the 33rd International Florida Artificial Intelligence Research Society Conference (FLAIRS'33), 557562. AAAI Press.

Atkinson, K.; Baroni, P.; Giacomin, M.; Hunter, A.; Prakken, H.; Reed, C.; Simari, G. R.; Thimm, M.; and Villata, S. 2017. Towards Artificial Argumentation. AI magazine 38(3):25-36.

Baroni, P.; Caminada, M.; and Giacomin, M. 2018. Abstract argumentation frameworks and their semantics. In Handbook of Formal Argumentation. College Publications. 159236.

Batens, D. 2007. A universal logic approach to adaptive logics. Logica Universalis 1(1):221-242.

Bench-Capon, T., and Dunne, P. 2007. Argumentation in artificial intelligence. Artificial Intelligence 171(10):619641.
Benferhat, S.; Dubois, D.; and Prade, H. 1997. Some syntactic approaches to the handling of inconsistent knowledge bases: A comparative study part 1: The flat case. Studia Logica 58(1):17-45.

Besnard, P., and Hunter, A. 2009. Argumentation based on classical logic. In Argumentation in Artificial Intelligence. Springer. 133-152.

Besnard, P., and Hunter, A. 2018. A review of argumentation based on deductive arguments. In Handbook of Formal Argumentation. College Publications. 437-484.

Besnard, P.; García, A.; Hunter, A.; Modgil, S.; Prakken, H.; Simari, G.; and Toni, F. 2014. Introduction to structured argumentation. Argument \& Computation 5(1):1-4.

Borg, A., and Straßer, C. 2018. Relevance in structured argumentation. In Lang, J., ed., Proceedings of the 27th International Joint Conference on Artificial Intelligence (IJCAI'18), 1753-1759. ijcai.org.

Borg, A. 2020. Assumptive sequent-based argumentation. Journal of Applied Logics-IfCoLog Journal of Logics and their Applications 7(3):227-294.

Caminada, M., and Amgoud, L. 2007. On the evaluation of argumentation formalisms. Artificial Intelligence 171(5):286-310.

Caminada, M.; Carnielli, W.; and Dunne, P. 2011. Semi-stable semantics. Journal of Logic and Computation 22(5):1207-1254.

Caminada, M. 2018. Rationality postulates: Applying argumentation theory for non-monotonic reasoning. In Handbook of Formal Argumentation. College Publications. 771795.

Cayrol, C. 1995. On the relation between argumentation and non-monotonic coherence-based entailment. In Proceedings of the 14th International Joint Conference on Artificial Intelligence (IJCAI'95), 1443-1448. Morgan Kaufmann.

Cerutti, F.; Gaggl, S. A.; Thimm, M.; and Wallner, J. P. 2017. Foundations of implementations for formal argumentation. Journal of Applied Logics-IfCoLog Journal of Logics and their Applications 4(8).

Corsi, E. A., and Fermüller, C. G. 2017. Logical argumentation principles, sequents, and nondeterministic matrices. In Proceedings of the 6th International Workshop on Logic, Rationality, and Interaction (LORI'17), volume 10455 of Lecture Notes in Computer Science, 422-437. Springer.

Corsi, E. A., and Fermüller, C. G. 2019. Connecting fuzzy logic and argumentation frames via logical attack principles. Soft Computing 23(7):2255-2270.

Čyras, K., and Toni, F. 2015. Non-monotonic inference properties for assumption-based argumentation. In Proceedings of the 3rd International Workshop on Theory and Applications of Formal Argument (TAFA'15), volume 9524 of Lecture Notes in Computer Science, 92-111. Springer.

Čyras, K., and Toni, F. 2016. ABA+: assumption-based argumentation with preferences. In Baral, C.; Delgrande, J.; and Wolter, F., eds., Proceedings of the 15th International Conference on Principles of Knowledge Representation and Reasoning (KR'16), 553-556. AAAI Press. 
da Costa, N. 1974. On the theory of inconsistent formal systems. Notre Dame Journal of Formal Logic 15(4):497510 .

D’Agostino, M., and Modgil, S. 2020. A fully rational account of structured argumentation under resource bounds. In Proceedings of the 29th International Joint Conference on Artificial Intelligence (IJCAI'20), 1841-1847. ijcai.org.

Dung, P. M.; Kowalski, R.; and Toni, F. 2009. Assumptionbased argumentation. In Argumentation in Artificial Intelligence. Springer. 199-218.

Dung, P. M. 1995. On the acceptability of arguments and its fundamental role in nonmonotonic reasoning, logic programming and n-person games. Artificial Intelligence 77(2):321-357.

Dunne, P., and Bench-Capon, T. 2003. Two party immediate response disputes: Properties and efficiency. Artificial Intelligence 149(2):221-250.

Gabbay, D. 1985. Theoretical foundations for nonmonotonic reasoning in expert systems. In Logics and models of concurrent systems. Springer. 439-457.

Gentzen, G. 1934. Untersuchungen über das logische Schließen I, II. Mathematische Zeitschrift 39:176-210, 405431.

Gorogiannis, N., and Hunter, A. 2011. Instantiating abstract argumentation with classical logic arguments: Postulates and properties. Artificial Intelligence 175(9-10):14791497.

Grossi, D. 2009. Doing argumentation theory in modal logic. Technical report, ILLC Technical Report PP-200924.

Grossi, D. 2011. Argumentation in the view of modal logic. In McBurney, P.; Rahwan, I.; and Parsons, S., eds., Proceedings of the 7th International Workshop on Argumentation in Multi-Agent Systems (ArgMAS'11), 190-208. Springer.

Heyninck, J., and Arieli, O. 2020. Simple contrapositive assumption-based frameworks. Journal of Approximate Reasoning 121:103-124.

Heyninck, J., and Straßer, C. 2021. A comparative study of assumption-based approaches to reasoning with priorities. Journal of Applied Logics-IfCoLog Journal of Logics and their Applications 8(3):737-808.

Kraus, S.; Lehmann, D.; and Magidor, M. 1990. Nonmonotonic reasoning, preferential models and cumulative logics. Artificial Intelligence 44(1):167 - 207.

Lehmann, D., and Magidor, M. 1992. What does a conditional knowledge base entail? Artificial Intelligence 55:160.

Li, Z.; Oren, N.; and Parsons, S. 2018. On the links between argumentation-based reasoning and nonmonotonic reasoning. In Proceedings of the 4th International Workshop on Theory and Applications of Formal Argument (TAFA'17), volume 10757 of Lecture Notes in Computer Science, 6785. Springer.

Łos, J., and Suzsko, R. 1958. Remarks on sentential logics. Indagationes Mathematicae 20:177-183.
Makinson, D. 1994. General patterns in nonmonotonic reasoning. In Gabbay, D.; Hogger, C.; and Robinson, J., eds., Handbook of Logic in Artificial Intelligence and Logic Programming, volume 3. Oxford Science Publications. 35-110. Makinson, D. 2003. Bridges between classical and nonmonotonic logic. Logic Journal of the IGPL 11(1):69-96.

Modgil, S., and Prakken, H. 2013. A general account of argumentation with preferences. Artificial Intelligence 195:361-397.

Modgil, S., and Prakken, H. 2018. Abstract rule-based argumentation. In Handbook of Formal Argumentation. College Publications. 287-364.

Rescher, N., and Manor, R. 1970. On inference from inconsistent premises. Theory and Decision 1:179-217.

Shoham, Y. 1988. Reasoning About Change: Time and Causation from the Standpoint of Artificial Intelligence. MIT Press.

Straßer, C., and Arieli, O. 2019. Normative reasoning by sequent-based argumentation. Journal of Logic and Computation 29(3):387-415.

Straßer, C., and Šešelja, D. 2010. Towards the prooftheoretic unification of dung's argumentation framework: an adaptive logic approach. Journal of Logic and Computation 21(2):133-156.

Straßer, C. 2014. Adaptive Logics for Defeasible Reasoning. Applications in Argumentation, Normative Reasoning and Default Reasoning, volume 38 of Trends in Logic. Springer.

Urquhart, A. 2001. Many-valued logic. In Gabbay, D., and Guenthner, F., eds., Handbook of Philosophical Logic, volume II. Kluwer. 249-295. Second edition.

Van De Putte, F. 2013. Default assumptions and selection functions: A generic framework for non-monotonic logics. In Castro, F.; Gelbukh, A.; and González, M., eds., Proceedings of the 12th Mexican International Conference on Advances in Artificial Intelligence and Its Applications (MICAI'13), volume 8265 of Lecture Notes in Computer Science. Springer.

Vesic, S. 2013. Identifying the class of maxi-consistent operators in argumentation. Journal of Artificial Intelligence Research 47:71-93. 